\title{
Bifurcation and stability of a two-tower system under wind-induced parametric, external and self-excitation
}

\author{
Daniele Zulli, Angelo Luongo*
}

DISAT - Università degli Studi dell'Aquila, 67100 L'Aquila (AQ), Italy

\begin{abstract}
A B S T R A C T
A two degree of freedom nonlinear system, describing the dynamics of two towers exposed to turbulent wind flow and linked by a nonlinear viscous device, is considered. The steady component of the wind is responsible for self excitation, while the turbulent part causes both parametric and external excitations, considered in a specific resonance condition. The possible occurrence of multiple Hopf bifurcations is taken into account. The periodic and quasi periodic solutions are studied after applying a perturbation scheme. The effect of the viscous device on the dynamics of the structure is analyzed, in order to investigate its effectiveness in mitigating the oscillations of the two indepen dent towers.
\end{abstract}

\section{Introduction}

Bifurcation and stability analysis are challenging issues for nonlinear self excited dynamic systems, particularly when more than one degree of freedom is present. For instance, multi parameter families of self excited autonomous systems, under a suitable combination of parameters, undergo multiple Hopf bifurcations [ $\left.\begin{array}{ll}1 & 3\end{array}\right]$, in which several degrees of freedom are involved in the post critical dynamics. As an example, this is the case of coupled structures subjected to steady wind, where, in addition to mono modal steady motions, multi modal stable oscillations appear both in non resonant and internally resonant [4 9] conditions. If self and parametric excitations are both present in multi degree of freedom (d.o.f.) systems, the dynamic response is even richer. This specific occurrence is the object of investigations in [10 12]. The dynamics under the concurrent presence of self and (mono or multi frequency) external excitation are of great interest too. In this regard, a 2 d.o.f. system is analyzed in [13]. In the field of machine dynamics, the contemporary occurrence of the three kinds of excitations, namely self, parametric and external excitations for 2 d.o.f. systems is studied in $[14,15]$.

In the civil engineering field, a 2 d.o.f. system describing the in plane and out of plane dynamics of an inclined cable, under steady wind and periodic support motion, is studied in [16]. There, the wind is responsible for self excitation, while the support motion, simulating vehicle traffic on the deck, causes external and parametric excitations.

Structures exposed to turbulent wind are typical systems where the three sources of excitations can interact, indeed the steady part of the flow is responsible for the self excitation, while the multi frequency unsteady flow causes parametric and external forces (see [17]). In this context, a single tower simultaneously subjected to self, parametric and external excitations, modeled as a 1 d.o.f. nonlinear system, was studied by the authors in [18]. There, the effects of the interactions

\footnotetext{
* Corresponding author.

E-mail addresses: daniele.zulli@univaq.it (D. Zulli), angelo.luongo@univaq.it (A. Luongo).
} 
of the three sources of excitation were analyzed, and the bifurcation diagrams in terms of the unfolding parameters (wind velocity and frequency detuning) were drawn.

In this paper, it is hypothesized that two towers, with different mechanical properties, are close enough to be linked by a purely viscous device, able to mitigate the wind induced oscillations that each tower would experience if it were unlinked. The objective of the study is to investigate the effectiveness of the viscous connection, as passive controller, both in linear and nonlinear regimes. To this end, a system constituted by two towers, subjected to turbulent multi frequency wind, is considered. In particular one of the two towers is assumed to be resonant with the turbulent wind, while the other tower, more stiff and not in resonance condition, is linked to the first tower by a nonlinear viscous device. The towers are multistory shear type frames modeled, on the same framework of [18], through a corresponding homogeneous scheme of continuous shear rods. After the application of a Galerkin projection, the resulting 2 d.o.f. coupled nonlinear system, in which the three kinds of excitations are concurrently present, is handled with the Multiple Scale Method (see [19]). A bifurcation analysis is carried out and the equilibrium solutions, representing steady (periodic or quasi periodic) oscillations, are analyzed in the space of the bifurcation parameters. Their stability is discussed, highlighting the beneficial influence of the viscous device on the response of the structure.

\section{The model}

\subsection{Continuous model}

Two multistory shear type towers of different square sections are exposed to unsteady wind, uniformly acting all along their heights. They are linked by a nonlinear viscous device at the tip. The dynamics of the structure are studied in the cross wind plane (see Fig. 1), by neglecting the curvature in the orthogonal direction, which is typically considered as a small imperfection.

In Fig. 1, $v_{1_{j}}(t)$ and $v_{2_{k}}(t)$ are the (time dependent) cross wind displacements of the $j$ th and $k$ th stories of the left and right tower, respectively. The inter story heights of the two towers are $h_{1}$ and $h_{2}$, and the dimensions of the cross sections are $B_{1} \times B_{1}$ and $B_{2} \times B_{2}$, respectively. The total height of both the towers is $\ell$.

A corresponding homogeneous model, constituted by two planar rods, undergoing purely shear strain (shear rods), clamped at one end and linked with the viscous device at the tip, is adopted (see Fig. 2). All geometrical and mechanical nonlinearities are neglected for the rods, so that the passive controller and the aerodynamic forces are the unique sources of nonlinearities of the system. This is a consequence of having linearized the kinematics, according to the fact that cantilever beams exhibit a weak nonlinear behavior (see, e.g. [20]). A discussion on the implications of the continuous modeling is carried out in [18], where additional details are given; here the model is briefly summarized.
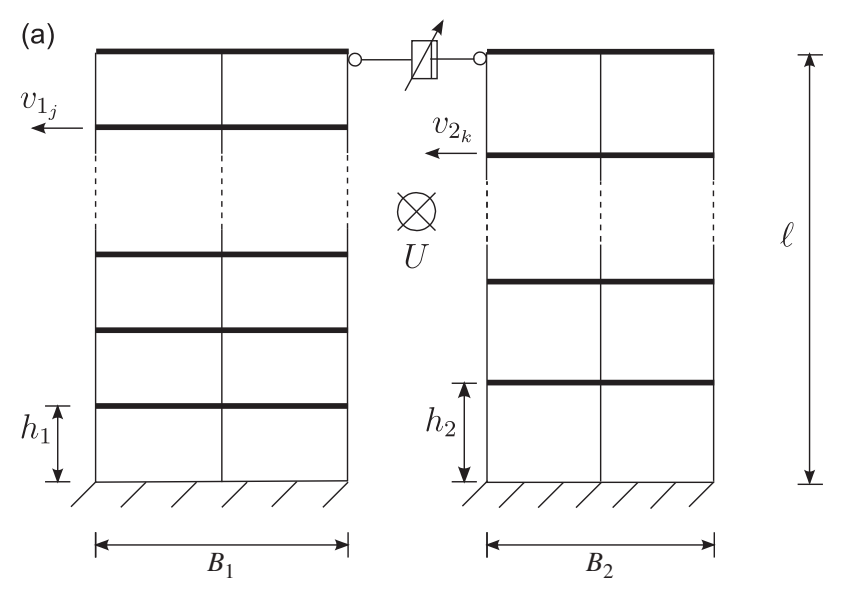

(b)

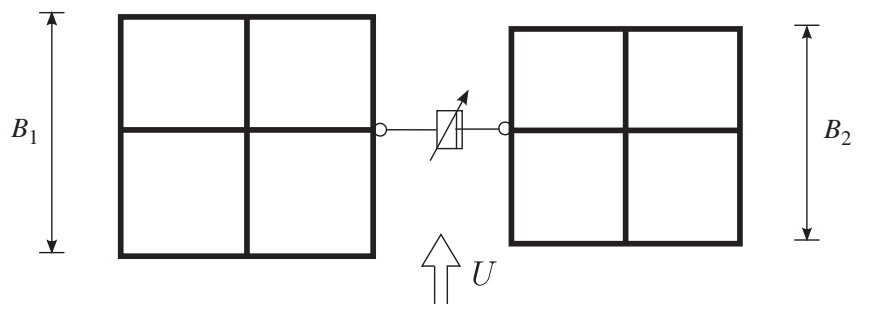

Fig. 1. Frontal (a) and top (b) views of the two towers exposed to the wind. 


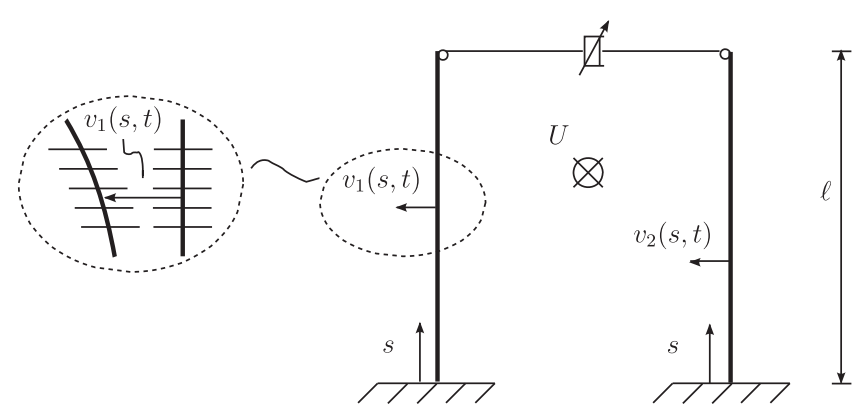

Fig. 2. Frontal view of the homogeneous model.

The shear rod is a one dimensional, inextensional, polar continuum, constituted by a flexible axis line and by rigid cross sections sliding in their plane. For the $i$ th rod, its deformed configuration is described by the transverse displacement of the axis line $v_{i}(s, t)(i=1,2)$ at abscissa $s$ and time $t$. The balance equation, expressing equilibrium in transverse direction, is

$$
T_{i}^{\prime}(s, t)+p_{i}(s, t)=0
$$

where $T_{i}(s, t)$ is the shear stress, $p_{i}(s, t)$ the load linear density, and a dash denotes space differentiation. By assuming a visco elastic constitutive law, obeying the Kelvin Voigt model, it follows that

$$
T_{i}(s, t)=G A_{i} v_{i}^{\prime}(s, t)+\eta_{i} \dot{v}_{i}^{\prime}(s, t)
$$

where $G A_{i}$ is the elastic shear stiffness, $\eta_{i}$ the internal damping coefficient and a dot denotes time differentiation. The load includes inertial, external damping and aerodynamic effects, so that

$$
p_{i}(s, t)=m_{i} v_{i}(s, t) \quad c_{i} \dot{v}_{i}(s, t)+p_{a_{i}}(s, t)
$$

$m_{i}$ being the mass per unit length, $c_{i}$ the external damping coefficient and $p_{a_{i}}(s, t)$ the linear density of aerodynamic forces, to be properly modeled. In conclusion, the balance equations read

$$
G A_{1} v_{i}^{\prime \prime}(s, t)+\eta_{i} \dot{v}_{i}^{\prime \prime}(s, t) \quad m_{i} v_{i}(s, t) \quad c_{i} \dot{v}_{i}(s, t)+p_{a_{i}}(s, t)=0, \quad i=1,2
$$

The value of the homogeneous shear stiffness for each tower is identified by equating the strain energy density of the discrete and the homogeneous models: it holds that $G A_{i}=n_{i}\left(12 E I_{i} / h_{i}^{2}\right)$, where $n_{i}$ is the number of columns in the generic story and $E I_{i}$ is the bending stiffness of each column, assumed the same throughout the tower.

The aerodynamic part of the load is related to the wind, which blows with a time dependent velocity $U(t)$. It applies lift forces, on the $i$ th $\operatorname{rod}(i=1,2)$, lying on the plane of the structure (see [21]), of the type

$$
p_{a_{i}}=\frac{1}{2} \rho U^{2} B_{i}\left[\mathcal{A}_{0}+\mathcal{A}_{1}\left(\frac{\dot{v}_{i}}{U}\right)+\mathcal{A}_{2}\left(\frac{\dot{v}_{i}}{U}\right)^{2}+\mathcal{A}_{3}\left(\frac{\dot{v}_{i}}{U}\right)^{3}\right]
$$

where $\mathcal{A}_{j}(j=0,1,2,3)$ are the aerodynamic coefficients relevant to square sections and $\rho$ the air mass density. Coherently with [18], the wind velocity is decomposed as $U(t)=\bar{U}+u(t)$, where $\bar{U}$ is the average steady component, and $u(t)$ is the (small) turbulent part, assumed periodic in time. Taylor's polynomial expansion provides the following expressions for the lift force on the two towers:

$$
p_{a_{i}}=\frac{1}{2} \rho B_{i}\left[C_{0}+C_{1} \dot{v}_{i}+C_{2} \dot{v}_{i}^{2}+C_{3} \dot{v}_{i}^{3}\right]
$$

where $C_{0}=\mathcal{A}_{0}\left(\bar{U}^{2}+2 \bar{U} u(t)\right), C_{1}=\mathcal{A}_{1}(\bar{U}+u(t)), C_{2}=\mathcal{A}_{2}$, and $C_{3}=\mathcal{A}_{3}\left(1 / \bar{U} u(t) / \bar{U}^{2}\right)$. Therefore, the aerodynamic force provides, by means of its steady part, terms which can be responsible for galloping and, by means of its turbulent part, periodic time dependent terms.

The boundary conditions for Eq. (4) are

$$
\begin{gathered}
v_{i}(0, t)=0 \\
G A_{i} v_{i}^{\prime}(\ell, t)+\eta_{i} \dot{v}_{i}^{\prime}(\ell, t)=\mp f\left(\dot{v}_{1}(\ell, t) \quad \dot{v}_{2}(\ell, t)\right)
\end{gathered}
$$

where the upper (lower) sign holds for the left (right) tower. Moreover, $f$ is the force exerted by the viscous device, depending on the relative velocity of the tip sections. It is supposed to have a constitutive law of the $f(\dot{\Delta})=\kappa_{1} \dot{\Delta}+\kappa_{3} \dot{\Delta}^{3}$ type, where $\dot{\Delta}:=\dot{v}_{1}(\ell) \quad \dot{v}_{2}(\ell)$, and where $\kappa_{1}$ and $\kappa_{3}$ are known parameters. It provides linear and cubic coupling terms in the system.

Non dimensional quantities are introduced here:

$$
s^{*}=\frac{s}{\ell}, \quad t^{*}=\omega_{1} t, \quad v_{i}^{*}=\frac{v_{i}}{\ell}, \quad \bar{U}^{*}=\frac{\bar{U}}{\bar{U}_{0}}, \quad u^{*}=\frac{u}{\bar{U}_{0}}
$$


where $\omega_{1}$ is the first natural frequency of the left beam and $\bar{U}_{0}$ is a reference value of the wind velocity, defined in the following subsection, whose meaning will be clear soon. The star will be dropped ahead for convenience of notation.

\subsection{Discrete model}

The system (4) (7) is discretized as a 2 d.o.f. system, via the Galerkin method, assuming as trial functions the first in plane mode for each rod, evaluated in the absence of wind, (external and internal) damping and viscous device. As extensively discussed in [18], the use of the sole first mode for each tower is justified by the effect of damping. According to the Rayleigh model adopted, the external viscous component (proportional to $c_{i}$ ) entails a decrement of the modal damping ratio with the mode number, while the internal viscous component (proportional to $\eta_{i}$ ) increases such a ratio (see the later Eq. $\left(11_{3,4}\right)$ ). Since the system is internally damped at a sufficient extent (as it generally happens in buildings), the higher modes weakly participate to the overall response. A numerical estimation of the contribution of the second mode is given in [18].

According to the previous discussion, it is assumed that $v_{1}(\mathrm{~s}, t)=x(t) \phi_{1}(s)$ and $v_{2}(s, t)=y(t) \phi_{2}(s)$, where $x(t), y(t)$ are the unknown (non dimensional) amplitudes of the tips of the two rods, and

$$
\phi_{i}(s)=\sin \left(\frac{\pi}{2} s\right)
$$

It should be noted that, since $\phi_{i}^{\prime}(1)=0$, the internal viscous force $\eta_{i} \dot{v}_{i}^{\prime}(\ell, t)$ at the boundary (Eq. (7)) does not produce work in the trial function, so that its contribution to the motion is lost. However, since $\eta_{i} \omega_{i} \ll G A_{i}$, it is negligible with respect to the elastic forces.

The two resulting second order, non homogeneous, time periodic, ordinary differential equations read

$$
\begin{aligned}
& x+\left(2 \xi_{1}(1 \quad \bar{U}) \quad b_{1} u(t)\right) \dot{x}+x+b_{2} \dot{x}^{2}+\left(\frac{b_{31}}{\bar{U}}+\frac{b_{32}}{\bar{U}^{2}} u(t)\right) \dot{x}^{3}+k_{1}(\dot{x} \quad \dot{y})+k_{3}(\dot{x} \quad \dot{y})^{3}=f_{1} \bar{U} u(t)+f_{3} \bar{U}^{2} \\
& y \quad\left(2 \xi_{2} \omega_{r} \quad c_{a_{2}} \bar{U} \quad b_{4} u(t)\right) \dot{y}+\omega_{r}^{2} y+b_{5} \dot{y}^{2}+\left(\frac{b_{61}}{\bar{U}}+\frac{b_{62}}{\bar{U}^{2}} u(t)\right) \dot{y}^{3} \quad k_{2}(\dot{x} \quad \dot{y}) \quad k_{4}(\dot{x} \quad \dot{y})^{3}=f_{2} \bar{U} u(t)+f_{4} \bar{U}^{2}
\end{aligned}
$$

where $\omega_{r}:=\omega_{2} / \omega_{1}$ is the ratio between the natural frequencies of the two rods; $\xi_{1}$ and $\xi_{2}$ are the structural modal damping ratios; the dot represents here differentiation with respect to the non dimensional time. The expressions of the coefficients of Eq. (10) are

$$
\begin{gathered}
\omega_{1}=\frac{\pi}{2 \ell} \sqrt{\frac{G A_{1}}{m_{1}}}, \quad \omega_{2}=\frac{\pi}{2 \ell} \sqrt{\frac{G A_{2}}{m_{2}}}, \quad \xi_{1}=\frac{\eta_{1} \omega_{1}}{2 G A_{1}}+\frac{c_{1}}{2 m_{1} \omega_{1}} \\
\xi_{2}=\frac{\eta_{2} \omega_{2}}{2 G A_{2}}+\frac{c_{2}}{2 m_{2} \omega_{2}}, \quad b_{1}=2 \xi_{1}, \quad b_{2}=\frac{4 \rho \mathcal{A}_{2} B_{1} \ell}{3 \pi m_{1}} \\
b_{31}=\frac{3 \rho \mathcal{A}_{3} B_{1} \ell^{2} \omega_{1}}{8 m_{1}}, \quad b_{32}=\quad b_{31}, \quad k_{1}=\frac{2 \kappa_{1}}{m_{1} \omega_{1}}, \quad k_{3}=\frac{2 \kappa_{3} \omega_{1}}{m_{1} \ell} \\
\bar{U}_{0}=\frac{4 m_{1} \omega_{1} \xi_{1}}{\rho B_{1} \mathcal{A}_{1}}, \quad \beta=\frac{B_{1}}{B_{2}}, \quad \alpha=\frac{m_{2}}{m_{1}}, \quad f_{1}=\frac{4 \rho B_{1} \mathcal{A}_{0} \bar{U}_{0}^{2}}{\pi \omega_{1}^{2} \ell m_{1}}, \quad f_{3}=\frac{f_{1}}{\alpha} \\
c_{a_{2}}=\frac{\beta}{\alpha} 2 \xi_{1}, \quad b_{4}=c_{a_{2}}, \quad b_{5}=\frac{\beta}{\alpha} b_{2}, \quad b_{61}=\frac{\beta}{\alpha} b_{31} \\
b_{62}=\frac{\beta}{\alpha} b_{32}, \quad k_{2}=\frac{k_{1}}{\alpha}, \quad k_{4}=\frac{k_{3}}{\alpha}, \quad f_{2}=\frac{f_{1}}{\alpha}, \quad f_{4}=\frac{f_{3}}{\alpha}
\end{gathered}
$$

The natural frequencies $\omega_{1}, \omega_{2}$ are assumed to be incommensurable, i.e. there is no internal resonance. The turbulent part is considered as periodic and constituted of its first two frequencies: $u(t)=u_{1} \sin (\Omega t)+u_{2} \sin (2 \Omega t)$, where $u_{1}$ and $u_{2}$ are two amplitudes and $\Omega$ is the fundamental frequency. If the frequency of the turbulence is assumed close to $\Omega_{c}:=1$, the external excitation becomes 1:1 resonant and the parametric excitation becomes 1:2 resonant with the left tower.

From Eq. $\left(10_{1}\right)$ it follows that, when the non dimensional wind velocity assumes the critical value $\bar{U}=\bar{U}_{c}:=1$ and $u(t)=0$, the total damping (i.e. structural plus aerodynamic) of the left tower vanishes. Therefore $\bar{U}_{0}$ in Eq. $\left(8_{4}\right)$ represents the dimensional critical (Hopf) value of the mean velocity, whose expression is shown in Eq. $\left(11_{11}\right)$, when this tower is unlinked. Since the coupling of the viscous device is assumed small, it is expected that the critical velocity of the two tower system is close to 1 , but hopefully higher. Eq. $\left(10_{2}\right)$ shows that, due to the different mechanical and geometrical properties of the two sub systems, the right tower undergoes a Hopf bifurcation at a (non dimensional) velocity value close to $\bar{U}_{c_{2}}:=2 \xi_{2} \omega_{r} / c_{a_{2}}$. If $\bar{U}_{c_{2}} \neq 1$ (non interaction case), then the passive controller acts as if it connected the left tower to the ground, so that its beneficial effects are quite obvious. In contrast, the most interesting case occurs when the right tower bifurcates at a critical value $\bar{U}_{c_{2}}=1$ (interaction case), i.e. when a more degenerate non resonant double Hopf 
bifurcation manifests itself for the coupled system. The perfect coincidence (for the unlinked system) occurs when $\xi_{2}=\xi_{2_{c}}:=c_{a_{2}} / 2 \omega_{r}$. To analyze this codimension two bifurcation, in conjunction with external/parametric excitation, three bifurcation parameters are necessary. They are chosen as the velocity $\bar{U}$, the damping ratio $\xi_{2}$ and the forcing frequency $\Omega$. The objective is to study the behavior of the system in the space of the bifurcation parameters in the neighborhood of the critical point $\left(\bar{U}_{c}, \xi_{2_{c}}, \Omega_{c}\right)=\left(1, c_{a_{2}} / 2 \omega_{r}, 1\right)$. The remaining parameters (including $\left.\xi_{1}\right)$ are taken as fixed auxiliary parameters.

\section{The multiple scale analysis}

A multiple scale perturbation analysis is carried out in Eqs. (10), to build up the bifurcation equations governing the long time motion on the Center Manifold [1]. Since this manifold, in the double Hopf bifurcation under study, is four dimensional, the 2 d.o.f. system (10) cannot be further reduced. However, by exploiting the capability of the Multiple Scale Method to filter the fast dynamics, bifurcation equations for the slow flow are expected, already in normal form, whose analysis is much more easier than the original equations (10).

A dimensionless small parameter $\varepsilon$ is introduced and increments of the critical parameters are defined as

$$
\begin{aligned}
& \bar{U}=1+\varepsilon V \\
& \xi_{2}=\xi_{2_{c}} \quad \varepsilon \zeta \\
& \Omega=1+\varepsilon \sigma
\end{aligned}
$$

with $\mathcal{O}(V)=\mathcal{O}(\zeta)=\mathcal{O}(\sigma)=1 ; V$ will be referred to as the main bifurcation parameter, $\zeta$ the splitting and $\sigma$ the detuning parameter. Moreover the dependent variables $(x, y)$ and the coefficient of Eqs. (10) are rescaled as follows: $(x, y)=\varepsilon^{1 / 2}(\hat{x}, \hat{y})$; $\left(b_{1}, b_{4}\right)=\varepsilon\left(\hat{b}_{1}, \hat{b}_{4}\right) ;\left(b_{2}, b_{5}\right)=\varepsilon^{1 / 2}\left(\hat{b}_{2}, \hat{b}_{5}\right) ;\left(k_{1}, k_{2}\right)=\varepsilon\left(\hat{k}_{1}, \hat{k}_{2}\right) ;\left(f_{1}, f_{2}, f_{3}, f_{4}\right)=\varepsilon^{3 / 2}\left(\hat{f}_{1}, \hat{f}_{2}, \hat{f}_{3}, \hat{f}_{4}\right)$. The other coefficients are of order 1 . This means that linear damping, external and parametric excitation, as well as the nonlinearities are ordered so that they appear, all together, at the highest order perturbation equations here considered.

\subsection{Perturbation equations}

To apply the Multiple Scale Method, the dependent variables are also expanded as

$$
\left\{\begin{array}{l}
\hat{x} \\
\hat{y}
\end{array}\right\}=\left\{\begin{array}{l}
x_{0} \\
y_{0}
\end{array}\right\}+\varepsilon\left\{\begin{array}{l}
x_{1} \\
y_{1}
\end{array}\right\}
$$

After introducing two independent time scales $t_{0}:=t$ and $t_{1}:=\varepsilon t$, the derivative with respect to the time assumes the expression $d / d t=d_{0}+\varepsilon d_{1}$, where $d_{i}:=\partial / \partial t_{i}$. As a consequence, the perturbation equations, divided by $\varepsilon^{1 / 2}$, read (omitting the hats)

$$
\begin{aligned}
& \mathcal{O}\left(\varepsilon^{0}\right):\left\{\begin{array}{l}
d_{0}^{2} x_{0}+x_{0}=0 \\
d_{0}^{2} y_{0}+\omega_{r}^{2} y_{0}=0
\end{array}\right.
\end{aligned}
$$

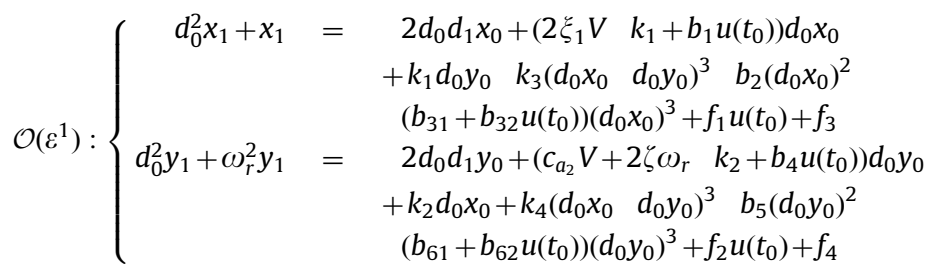

Eq. $\left(14_{1}\right)$ admits the following solution:

$$
\left\{\begin{array}{l}
x_{0} \\
y_{0}
\end{array}\right\}=\left\{\begin{array}{l}
A_{1}\left(t_{1}\right) \exp \left(\mathrm{i} t_{0}\right) \\
A_{2}\left(t_{1}\right) \exp \left(\mathrm{i} \omega_{r} t_{0}\right)
\end{array}\right\}+c c
$$

where cc denotes the complex conjugate, $\mathrm{i}$ is the imaginary unit and $A_{1}, A_{2}$ are unknown complex amplitudes. In terms of the detuning parameter $\sigma$, the turbulent part can be written as

$$
u\left(t_{0}\right)=\frac{1}{2} u_{1} \mathrm{i} \exp \left(\mathrm{i}(1+\varepsilon \sigma) t_{0}\right)+\frac{1}{2} u_{2} \mathrm{i} \exp \left(2 \mathrm{i}(1+\varepsilon \sigma) t_{0}\right)+\mathrm{cc}
$$

By substituting Eqs. (15) and (16) in Eq. $\left(14_{2}\right)$ and by zeroing the secular terms which arise in the right hand side, a set of differential equations in $A_{1}, A_{2}$ is obtained. Hence, by coming back to the true (non dimensional) time $t$, this set of equations provides the amplitude modulation equations (AME). They read as

$$
\dot{A}_{1}=\frac{1}{2}\left(2 \xi_{1} V \quad k_{1}\right) A_{1} \quad 3 k_{3} \omega_{r}^{2} A_{1} A_{2} \bar{A}_{2} \quad \frac{3}{2}\left(k_{3}+b_{31}\right) A_{1}^{2} \bar{A}_{1} \quad \frac{f_{1} u_{1}}{4} e^{\mathrm{i} \sigma t}+\mathrm{i} \frac{b_{1}}{4} u_{2} \bar{A}_{1} e^{2 \mathrm{i} \sigma t}+\mathrm{i} \frac{b_{32}}{4} u_{2} A_{1}^{3} e^{-2 \mathrm{i} \sigma t} \quad \mathrm{i} \frac{3}{4} b_{32} u_{2} A_{1} \bar{A}_{1}^{2} e^{2 \mathrm{i} \sigma t}
$$




$$
\dot{A}_{2}=\frac{1}{2}\left(c_{a_{2}} V+2 \zeta \omega_{r} \quad k_{2}\right) A_{2} \quad 3 k_{4} A_{1} A_{2} \bar{A}_{1} \quad \frac{3}{2}\left(b_{61}+k_{4}\right) \omega_{r}^{2} A_{2}^{2} \bar{A}_{2}
$$

It is worth noticing that the turbulent component provides external excitation by its component of amplitude $u_{1}$, and parametric excitation (both linear and cubic) by its component of amplitude $u_{2}$. Eqs. (17) also give the (split) critical values of the main parameter, as a first order perturbation of the (coalescent) double Hopf value $\bar{U}_{c}=1$. By zeroing the coefficients of the linear part of the right member, it follows that the increments in the critical wind velocity are $V_{c_{1}}=k_{1} / 2 \xi_{1}, V_{c_{2}}=\left(\begin{array}{ll}k_{2} & 2\end{array}\right.$ $\left.\zeta \omega_{r}\right) / c_{a_{2}}$. They are due to the passive controller (namely, its linear characteristic) and to the splitting parameter.

The polar form of Eqs. (17), obtained posing $A_{1}:=\frac{1}{2} a_{1} e^{\mathrm{i} \vartheta_{1}}, A_{2}:=\frac{1}{2} a_{2} e^{\mathrm{i} \vartheta_{2}}, \varphi_{1}:=\sigma t \quad \vartheta_{1}$ and $\varphi_{2}:=\vartheta_{2}$ (and referred as polar amplitude modulation equations), is

$$
\begin{aligned}
& \dot{a}_{1}=\frac{1}{2}\left(2 \xi_{1} V \quad k_{1} \quad \frac{1}{2} b_{1} u_{2} \sin \left(2 \varphi_{1}\right)\right) a_{1} \quad \frac{3}{4} k_{3} \omega_{r}^{2} a_{1} a_{2}^{2} \quad\left(\frac{3}{8} k_{3}+\frac{3}{8} b_{31} \quad \frac{b_{32}}{4} u_{2} \sin \left(2 \varphi_{1}\right)\right) a_{1}^{3} \quad \frac{f_{1}}{2} u_{1} \cos \varphi_{1} \\
& \dot{a}_{2}=\frac{1}{2}\left(c_{a_{2}} V \quad k_{2}+\zeta \omega_{r}\right) a_{2} \quad \frac{3}{4} k_{4} a_{1}^{2} a_{2} \quad \frac{3}{8} \omega_{r}^{2} a_{2}^{3}\left(k_{4}+b_{61}\right) \\
& a_{1} \dot{\varphi}_{1}=\left(\sigma \frac{b_{1}}{4} u_{2} \cos \left(2 \varphi_{1}\right)\right) a_{1}+\frac{b_{32}}{8} u_{2} \cos \left(2 \varphi_{1}\right)+\frac{f_{1}}{2} u_{1} \sin \varphi_{1} \\
& a_{2} \dot{\varphi}_{2}=0
\end{aligned}
$$

The variable $\varphi_{2}$ turns out to be worthless, being Eq. $\left(18_{4}\right)$ uncoupled and trivial.

Eqs. (17) suggest the following remarks. (a) The analysis includes the case in which the right tower possess a critical velocity greater than 1 (simple Hopf bifurcation case). Indeed, by letting $A_{2}=0$, Eq. $\left(17_{1}\right)$ is identically satisfied and Eq. $\left(17_{2}\right.$ ) (or, in polar form, Eqs. $\left(18_{1,3}\right)$ ) governs the system. Therefore, mono modal solutions $A_{1} \neq 0, A_{2}=0$, which are going to be analyzed in the next subsection, can also be considered as the unique solutions of this non interaction case. (b) The turbulence directly affects the left tower only, since $u_{1}$ and $u_{2}$ do not appear in Eq. $\left(18_{2}\right)$; however, due to the coupling term $k_{4}$ in Eq. $\left(18_{2}\right)$, the right tower is excited too. (c) If the towers are unlinked $\left(k_{i}=0, i=1, \ldots, 4\right)$, then, at the leading order of the asymptotic solution, the right tower is insensitive to turbulence. (d) Finally, if $A_{2}=0$ is considered for the unlinked system, the case of single tower, already studied in [18], is recovered.

\subsection{Fixed points analysis}

The fixed points of Eq. (18), obtained by letting $\dot{a}_{1}=\dot{a}_{2}=\dot{\varphi}_{1}=\dot{\varphi}_{2}=0$, represent steady state (periodic or quasi periodic) oscillations of the rods. Here, analytical expressions of them are sought.

In the absence of turbulence $\left(u_{1}=u_{2}=0\right)$, only self excitation is present and the non resonant double Hopf bifurcation is obtained (see [4]). Just Eq. $\left(18_{1,2}\right)$ are interesting, the phase $\varphi_{1}$ being a slave variable. In this case, in addition to the trivial solution $a_{1}=a_{2}=0$, indicated as $\mathrm{O}$, the classical mono modal galloping responses occur. One of them, indicated as I, is the following:

$$
\begin{gathered}
a_{1 e}=2 \sqrt{\frac{2 \xi_{1} V \quad k_{1}}{3\left(b_{31}+k_{3}\right)}} \\
a_{2 e}=0
\end{gathered}
$$

which occurs when $V \geq k_{1} / 2 \xi_{1}, \forall \zeta$. It represents the amplitude of periodic oscillations of the left tower, while the right tower experiences oscillations of higher order magnitude. It is worth noticing how the linear coefficient $k_{1}$ of the viscous device modifies the origin of the bifurcated branch, while the cubic coefficient $k_{3}$ modifies its amplitude. In particular, in case of purely nonlinear viscous device of hardening type $\left(k_{1}=0, k_{3}>0\right)$ and $b_{31}>0$, the mono modal galloping curve is always underneath the corresponding branch of the unlinked tower. Its stability is ruled by the sign of the real part of the eigenvalues of the Jacobian matrix, that read

$$
\lambda_{1,2}=\frac{1}{16}\left(3 a_{1 e}^{2}\left(3\left(b_{31}+k_{3}\right)+2 k_{4}\right)+4\left(V\left(2 \xi_{1}+c_{a_{2}}\right)+k_{1}+k_{2} \quad 2 \zeta \omega_{r}\right) \pm \sqrt{ }\left(a_{1 e}^{2}\left(9\left(b_{31}+k_{3}\right) \quad 6 k_{4}\right) \quad 4\left(\left(2 \xi_{1} c_{a_{2}}\right) V \quad k_{1}+k_{2} \quad 2 \zeta \omega_{r}\right)\right)^{2}\right)
$$

A second mono modal solution, indicated as II, is

$$
\begin{gathered}
a_{1 e}=0 \\
a_{2 e}=2 \sqrt{\frac{c_{a_{2}} V \quad k_{2}+2 \zeta \omega_{r}}{3 \omega_{r}^{2}\left(b_{61}+k_{4}\right)}}
\end{gathered}
$$

and occurs when $V \geq\left(\begin{array}{ll}k_{2} & 2 \zeta \omega_{r}\end{array}\right) / c_{a_{2}}$. It represents the amplitude of the periodic oscillations of the right tower, while the left tower undergoes oscillations of higher order magnitude. Its stability is governed by the following eigenvalues of the Jacobian matrix:

$$
\begin{aligned}
& \lambda_{1,2}=\frac{1}{16}\left(3 a_{2 e}^{2} \omega_{r}^{2}\left(3 b_{61}+2 k_{3}+3 k_{4}\right) \quad 4 V\left(2 \xi_{1}+c_{a_{2}}\right)+4 k_{1}+4 k_{2} \quad 8 \zeta \omega_{r}\right. \\
& \left.\left. \pm \sqrt{\left(3 a _ { 2 e } ^ { 2 } \omega _ { r } ^ { 2 } \left(3 b_{61}\right.\right.} 2 k_{3}+3 k_{4}\right)+4\left(\begin{array}{llll}
2 \xi_{1} V & c_{a_{2}} V & \left.k_{1}+k_{2}\right) & 8 \zeta \omega_{r}
\end{array}\right)^{2}\right)
\end{aligned}
$$


A bi modal solution, indicated as III, is

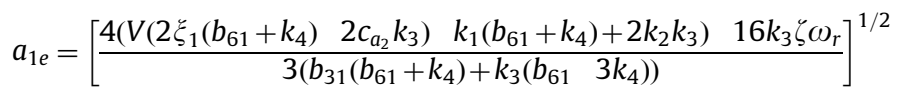

$$
\begin{aligned}
& a_{2 e}=\left[\frac{4\left(V\left(c_{a_{2}}\left(b_{31}+k_{3}\right) 4 \xi_{1} k_{4}\right) k_{2}\left(b_{31}+k_{3}\right)+2 k_{1} k_{4}\right)+8 \zeta \omega_{r}\left(b_{31}+k_{3}\right)}{3 \omega_{r}^{2}\left(b_{31}\left(b_{61}+k_{4}\right)+k_{3}\left(b_{61} 3 k_{4}\right)\right)}\right]^{1 / 2}
\end{aligned}
$$

It represents the amplitude of coupled quasi periodic oscillations of the two towers. The corresponding eigenvalues of the Jacobian matrix are not reported here for brevity.

The effect of turbulence will be addressed considering first the external contribution only $\left(u_{1} \neq 0, u_{2}=0\right)$, then considering the parametric contribution only $\left(u_{1}=0, u_{2} \neq 0\right)$ and finally the contemporary presence of the two contribu tions $\left(u_{1} \neq 0, u_{2} \neq 0\right)$. In every cases, the relevant equations are Eq. $\left(18_{1,2,3}\right)$.

In the first case $\left(u_{1} \neq 0, u_{2}=0\right)$, two nonlinear algebraic equations for steady points can be drawn in the following way: $\cos \varphi_{1}$ is obtained by zeroing the right hand side of Eq. $\left(18_{1}\right), \sin \varphi_{1}$ is obtained by zeroing the right hand side of Eq. $\left(18_{3}\right)$, and then the variable $\varphi_{1}$ is condensed using the relation $\cos ^{2} \varphi_{1}+\sin ^{2} \varphi_{1}=1$. The resulting equation, where only $a_{1}$ and $a_{2}$ appear, is the following:

$$
\frac{a_{1}^{2}\left(\left(3 a_{1}^{2}\left(b_{31}+k_{3}\right)+6 k_{3} \omega_{r}^{2} a_{2}^{2} \quad 8 \xi_{1} V+4 k_{1}\right)^{2}+64 \sigma^{2}\right)}{16 f_{1}^{2} u_{1}^{2}}=1
$$

Eq. (24) is sided by the equation obtained by setting the right hand side of Eq. (182) equal to zero.

In the second case $\left(u_{1}=0, u_{2} \neq 0\right)$, the corresponding two nonlinear algebraic equations can be obtained as follows: $\sin \left(2 \varphi_{1}\right)$ is obtained by zeroing the right hand side of Eq. $\left(18_{1}\right), \cos \left(2 \varphi_{1}\right)$ is obtained by zeroing the right hand side of Eq. $\left(18_{3}\right)$, and then, again, the variable $\varphi_{1}$ is condensed using the relation $\cos ^{2}\left(2 \varphi_{1}\right)+\sin ^{2}\left(2 \varphi_{1}\right)=1$. The resulting equation, where only $a_{1}$ and $a_{2}$ appear, is the following:

$$
\frac{\left(3 a_{1}^{2}\left(b_{31}+k_{3}\right)+6 k_{3} \omega_{r}^{2} a_{2}^{2} 8 \xi_{1} V+4 k_{1}\right)^{2}}{4 u_{2}^{2}\left(b_{1} b_{32} a_{1}^{2}\right)^{2}}+\frac{256 \sigma^{2}}{4 u_{2}^{2}\left(b_{32} a_{1}^{2} \quad 2 b_{1}\right)^{2}}=1
$$

As in the previous case, Eq. (25) is sided by the equation obtained by setting the right hand side of Eq. ( $18_{2}$ ) equal to zero.

In the third case $\left(u_{1} \neq 0, u_{2} \neq 0\right)$, it is not possible to apply a corresponding procedure, since terms of type $\sin 2 \varphi_{1}$, $\cos 2 \varphi_{1}, \sin \varphi_{1}, \cos \varphi_{1}$ are concurrently present. In this case, it means that it is necessary to solve a nonlinear system of three equations, considering also $\varphi_{1}$, besides $a_{1}$ and $a_{2}$.

Solutions of the systems coming from Eqs. (24) and (25), respectively, and from the generic case are sought by numerical procedures and are discussed in the following section.

\section{Numerical results}

The following numerical values are used for a case study. For the left tower (which is the same one that was analyzed in [18]): the cross section is $B_{1}=16 \mathrm{~m}$ wide, the total stiffness of the single story is $E I_{1}=115318000 \mathrm{~N} \mathrm{~m}^{2}$, the mass per unit length is $m_{1}=4737 \mathrm{~kg} / \mathrm{m}$, the damping ratio is $\xi_{1}=0.5$ percent (corresponding to $\eta_{1}=128513 \mathrm{~N} \mathrm{~s}, c_{1}=34.8675 \mathrm{~N} \mathrm{~s} / \mathrm{m}^{2}$ ). For the right tower: $B_{2}=15 \mathrm{~m}, E I_{2}=229736000 \mathrm{~N} \mathrm{~m}^{2}, m_{2}=3265 \mathrm{~kg} / \mathrm{m}$. The height of the two towers and the inter story height are assumed the same for the two towers, namely $\ell=36 \mathrm{~m}$ and $h=4 \mathrm{~m}$. The aerodynamic coefficients $\mathcal{A}_{i}, i=0, \ldots, 3$ are taken from [17] for the square cross section: $\mathcal{A}_{0}=0.0297, \mathcal{A}_{1}=0.9298, \mathcal{A}_{2}=0.2400, \mathcal{A}_{3}=7.6770$. The air mass density is $\rho=1.25$ $\mathrm{kg} / \mathrm{m}^{3}$. The (dimensional) natural frequencies of the towers are found to be $\omega_{1}=5.89 \mathrm{rad} / \mathrm{s}$ and $\omega_{2}=10.01 \mathrm{rad} / \mathrm{s}$, respectively, so that $\omega_{r}=1.7$. The (dimensional) critical wind velocity for the unlinked left tower assumes the value $\bar{U}_{0}=30 \mathrm{~m} / \mathrm{s}$ and the critical value for the damping ratio of the right tower is $\xi_{2_{c}}=0.4$ percent. The linear coefficient of the viscous device has the value $\kappa_{1}=100 \mathrm{~kg} / \mathrm{s}$ (corresponding to $k_{1}=1.99 \times 10^{-4}$ ), while different values are used for the cubic coefficient. In particular, a distinction between the categories of the viscous damping is made with respect to the value of the ratio $v:=k_{3} / b_{31}$, namely if $0<v \leq 1$ the device is hard, if $v>1$ it is very hard. In this case study, the following values are considered: for a hard device $\kappa_{3}=12.5 \mathrm{~kg} \mathrm{~s} / \mathrm{m}^{2}$ (corresponding to $k_{3}=1.12$ and to $v=0.36$ ), for a very hard device $\kappa_{3}=112.5 \mathrm{~kg} \mathrm{~s} / \mathrm{m}^{2}$ (corresponding to $k_{3}=10.8$ and to $v=3.25$ ). Soft devices, i.e. $\kappa_{3}<0 \mathrm{~kg} \mathrm{~s} / \mathrm{m}^{2}$ (corresponding to $v<0$ ), have been also considered, leading to disadvantageous results in comparison with the case of unlinked towers and therefore not reported here.

In the following subsections four different wind loads are considered separately, namely: (a) non turbulent wind $\left(u_{1}=0, u_{2}=0\right)$ for which only self excitation is triggered at $V \geq 0$; (b) turbulent wind, producing self and simultaneous external excitation $\left(u_{1} \neq 0, u_{2}=0\right)$; (c) turbulent wind, causing self and parametric excitation $\left(u_{1}=0, u_{2} \neq 0\right)$; (d) turbulent wind, entailing simultaneous self , parametric and external excitations $\left(u_{1} \neq 0, u_{2} \neq 0\right)$.

\subsection{Non turbulent wind}

The behavior charts of the system, relevant to non turbulent wind, are shown in Fig. 3 for both hard and very hard viscous devices. According to a common representation of bifurcation analysis (see e.g. [1]), these charts organize the 

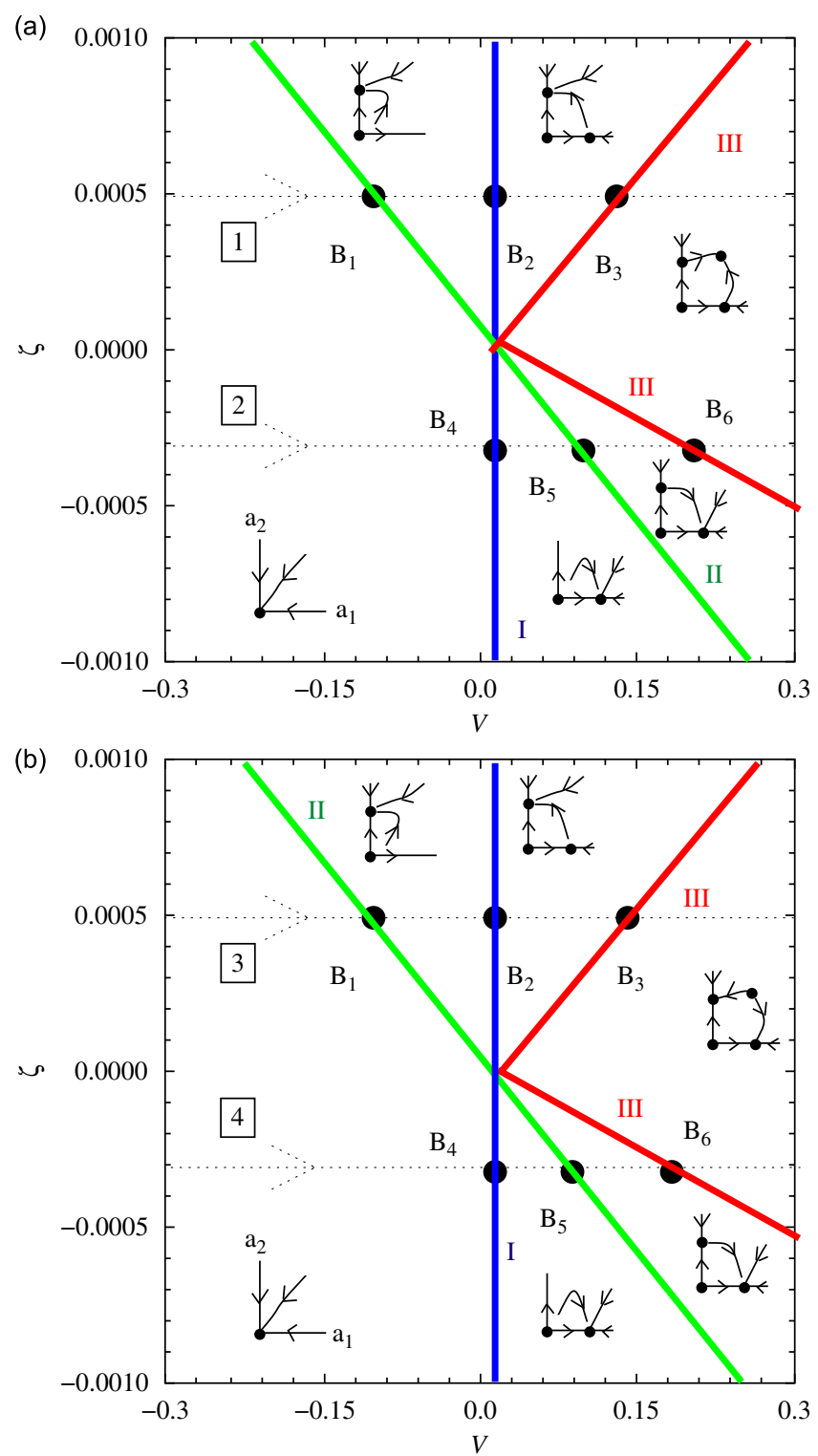

Fig. 3. Stability diagram for non-turbulent wind; viscous device: (a) hard, (b) very hard.

parameter space in regions (sub families of equivalent physical systems) in which the same qualitative dynamics occur, the regions being separated by bifurcation loci at which the behavior abruptly change. For each sub family, the relevant dynamics are displayed by a sketch of the $\left(a_{1}, a_{2}\right)$ phase plane for Eqs. $\left(18_{1,2}\right)$. From these sketches it emerges that (periodic) mono modal solutions (equilibrium points on the axes) and (quasi periodic) bimodal solutions (equilibrium points not belonging to the axes) do not exist in the whole plane, but only in some regions. Moreover, the orientation of the orbits denotes stability or instability of the motion. The solution $O$ exists in the whole plane $(V, \zeta)$. The lines labeled with I and II are the boundary limits for the existence of the mono modal solutions I and II, respectively; the lines indicated as III represent the boundary limits for solution III.

The significant difference between the hard and very hard cases is related to the stability of the solutions inside the region of existence of the bi modal III: in the hard case the bi modal motion is stable and the mono modal motions are unstable; the opposite happens for very hard viscous devices. In particular, starting from the condition of Fig. 3a, if the value of $\kappa_{3}$ is gradually increased, with all the other parameters fixed, the upper line III of Fig. 3a rotates clockwise, while the lower line III rotates counterclockwise, reducing the amplitude of the domain of existence of the solution III. For a specific value of $\kappa_{3}$ (corresponding to $v=1$ ), the two lines overlap. Further increasing $\kappa_{3}$ produces a separation of the two lines, allowing again the existence of the bi modal motion III, which is unstable. The two chosen values for $\kappa_{3}$ are those leading to the same amplitudes of the domain of existence of the solution III, in the two plots of Fig. 3. 

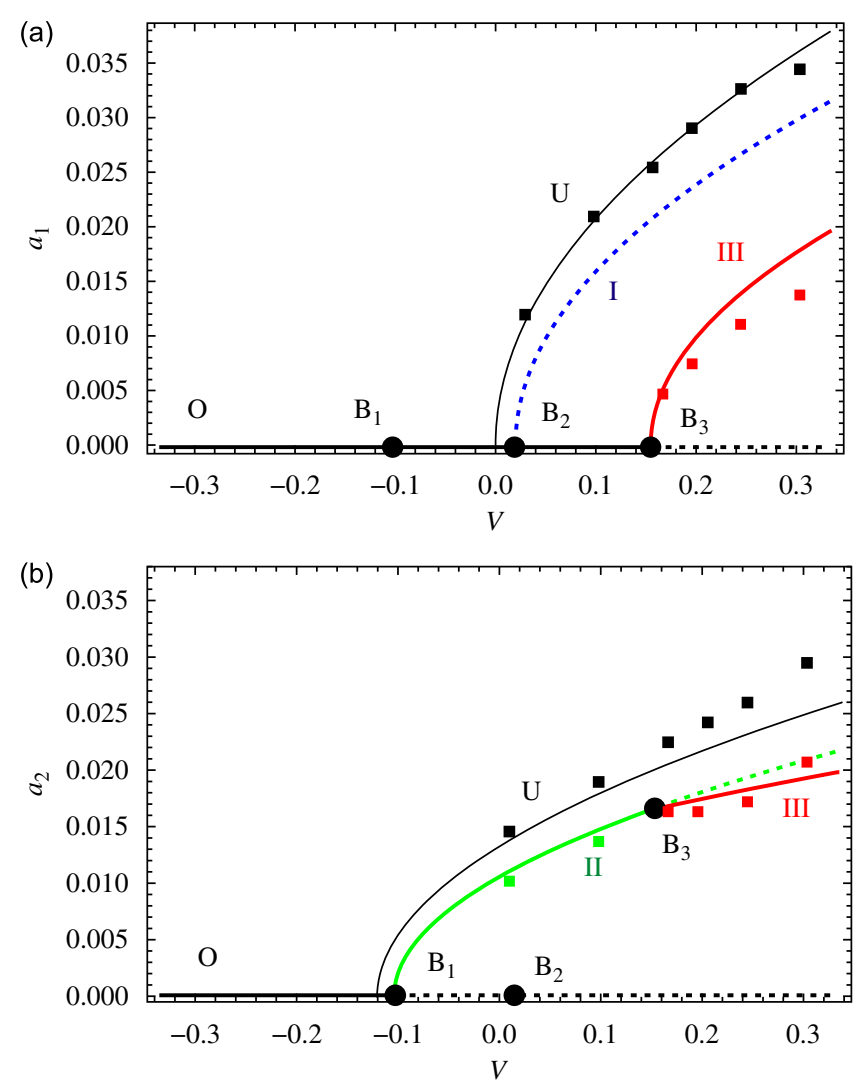

Fig. 4. Limit-cycle amplitudes $a_{1}$ (a) and $a_{2}$ (b) vs. $V$ for non-turbulent wind, hard viscous device and $\zeta \quad 0.0005$ (path 1 in Fig. $3 a$ ). (O) Trivial; (I) monomodal $a_{1}$; (II) mono-modal $a_{2}$; (III) bi-modal $\left(a_{1}, a_{2}\right)$; (U) unlinked towers; continuous line: stable; dashed line: unstable, boxes: numerical integrations of the original equations (10).

Path 1 in Fig. 3a is the trace of the section of the $\left(a_{k}, V, \zeta\right)$ bifurcation diagram, shown in Fig. 4, for $\zeta=0.0005$. In particular, for low values of $V$ (i.e. on the left of $B_{1}$ ), just the stable trivial solution $O$ exists. Increasing $V$, after the bifurcation point $\mathrm{B}_{1}$, two solutions exist: the trivial one $\mathrm{O}$, which becomes unstable, and the mono modal galloping II of the right tower, which is stable. It is worth noticing how the viscous device increases the actual critical wind velocity and decreases the amplitude of oscillations for the second tower $\left(a_{2}\right)$, in comparison with the unlinked tower case (thin line). Further increasing the value of $V$ leads to the bifurcation point $\mathrm{B}_{2}$, from where the other mono modal branch I emanates; it is unstable. Even in this condition, a strong advantage is produced by the device, since the left tower $\left(a_{1}\right)$ experiences oscillations of higher amplitude order, differently from the unlinked case (thin line). The bi modal branch, which is stable, starts from the bifurcation point $B_{3}$, and the oscillation amplitudes are always lower than those in the unlinked case. To check the reliability of the results obtained through the method of multiple scales, the amplitudes resulting from direct numerical integrations (variable step Runge Kutta method) of the original equations (10) are superimposed (filled boxes) in Fig. 4, showing good agreement. The numerical time laws of $x(t)$ and $y(t)$ for $V=0.3$, corresponding to the stable bi modal solution III, are shown in Fig. 5 as well, compared with the response of the unlinked towers.

The second path, corresponding to $\zeta=0.0003$ (line marked as 2 in Fig. 3a), is shown in Fig. 6 . The stable mono modal branch I bifurcates from the trivial solution at $\mathrm{B}_{4}$, and loses stability at $\mathrm{B}_{6}$; here the bi modal stable branch III emanates from. The other mono modal solution II is unstable and starts from $B_{5}$. When these amplitudes are compared, the beneficial effect of the viscous device is remarkable too.

A different behavior is obtained following the path of line 3 of Fig. 3b, i.e. for very hard devices, when $\zeta=0.0005$ (see Fig. 7). Here, after the bifurcation point $\mathrm{B}_{3}$, the bi modal branch III is unstable, while the two mono modal branches are stable. The mitigation of the oscillation, in comparison with the unlinked case, is even more evident along this path.

Following the path of line 4 of Fig. 3b, the bifurcation diagram of Fig. 8 is obtained where, again, the viscous device works well.

\subsection{Turbulent wind as external excitation}

When the wind is turbulent, a third parameter $\sigma$ (the detuning) must be taken into account, in addition to the two bifurcation parameters $V, \zeta$ previously considered. To simplify the analysis, the splitting damping parameter is fixed at the 

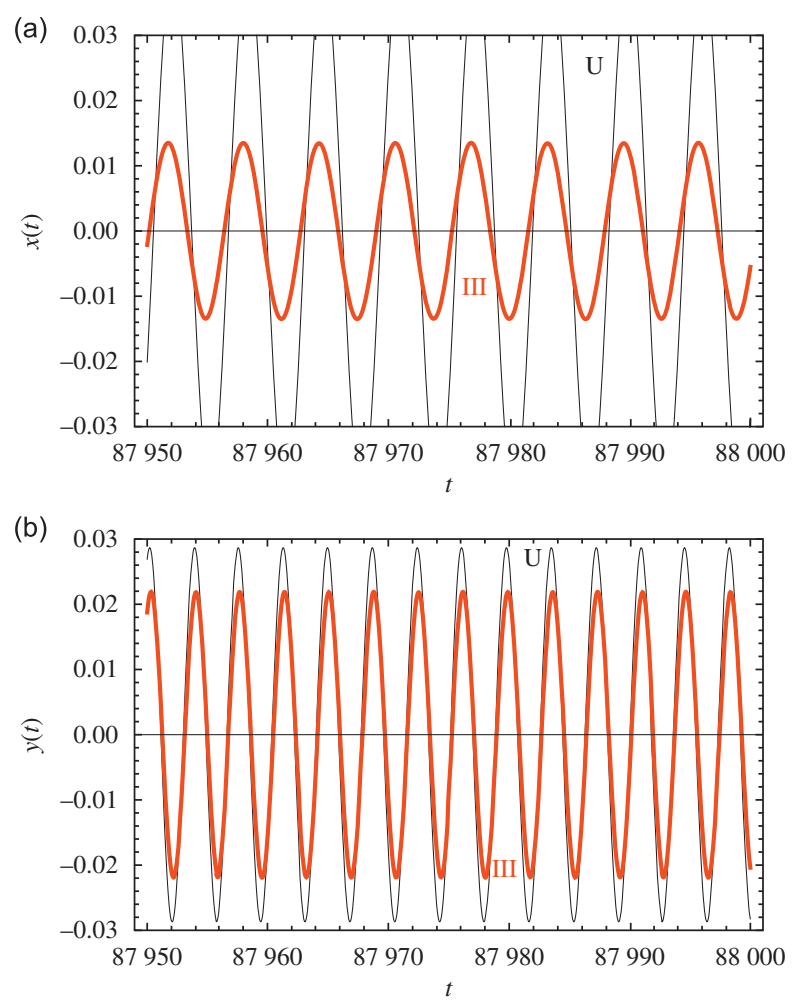

Fig. 5. Numerical integrations of Eq. (10): $x(t)(\mathrm{a})$ and $y(t)(\mathrm{b})$ for non-turbulent wind, hard viscous device and $V \quad 0.3, \zeta \quad 0.0005$. (III) Bi-modal; (U) unlinked towers.
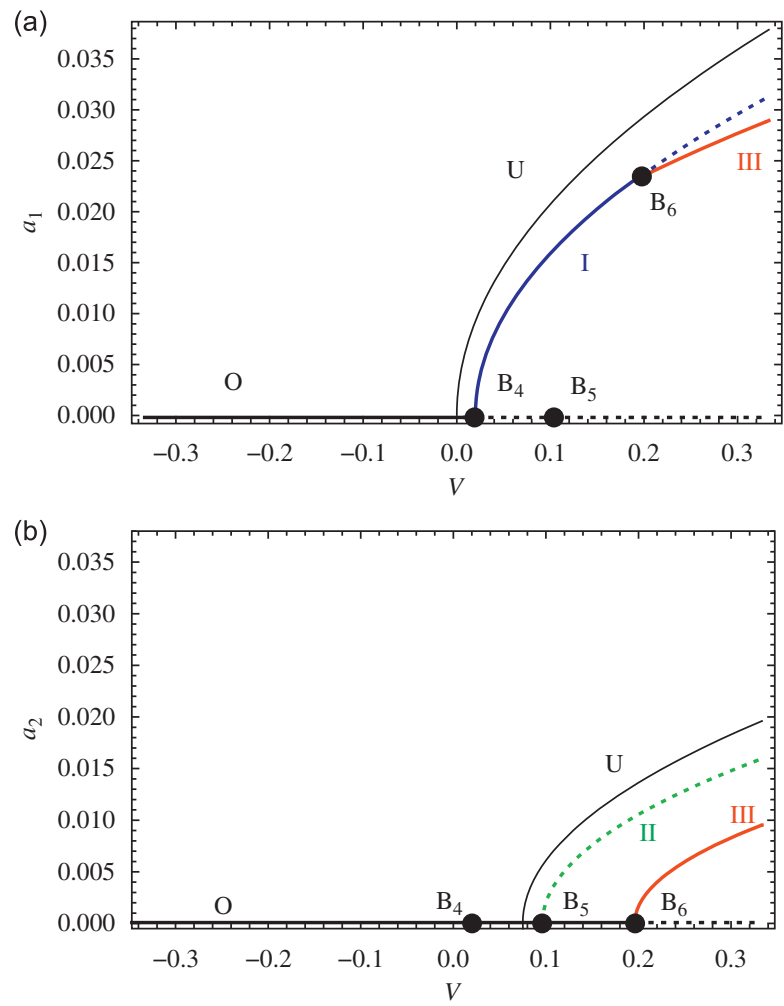

Fig. 6. Limit-cycle amplitudes $a_{1}$ (a) and $a_{2}$ (b) vs. $V$ for non-turbulent wind, hard viscous device and $\zeta \quad-0.0003$ (path 2 in Fig. $3 a$ ). (0) Trivial; (I) mono-modal $a_{1}$; (II) mono-modal $a_{2}$; (III) bi-modal $\left(a_{1}, a_{2}\right)$; (U) unlinked towers; continuous line: stable; dashed line: unstable. 
(a)
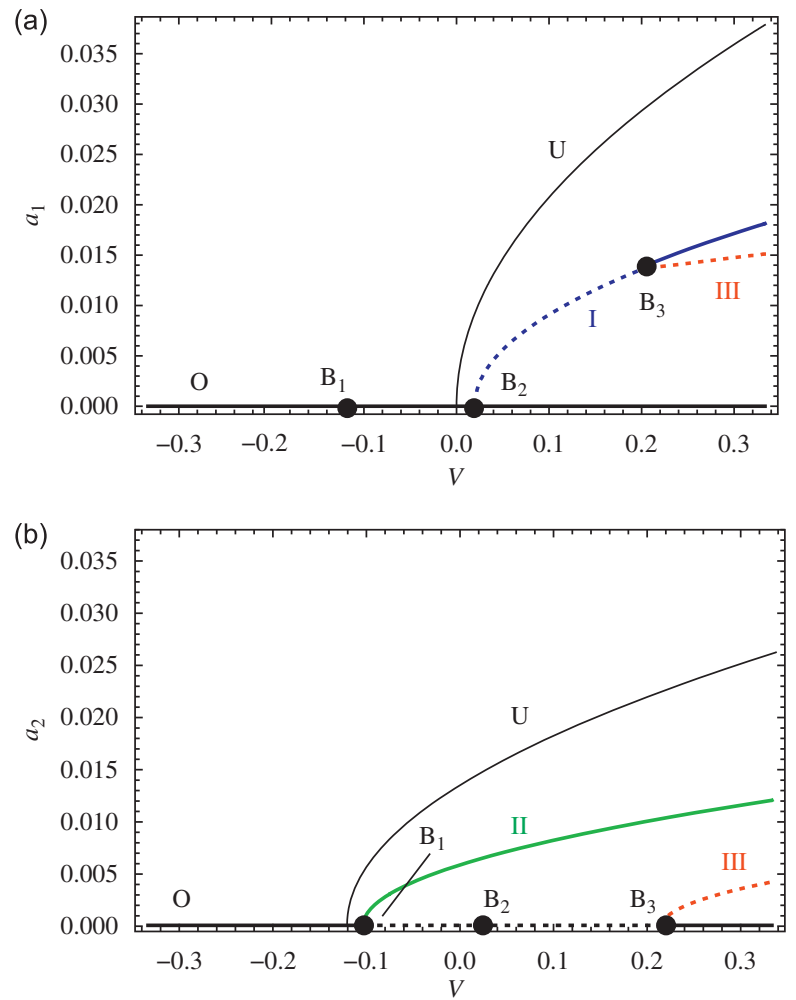

Fig. 7. Limit-cycle amplitudes $a_{1}$ (a) and $a_{2}$ (b) vs. $V$ for non-turbulent wind, very hard viscous device and $\zeta \quad 0.0005$ (path 3 in Fig. $3 \mathrm{~b}$ ). (0) Trivial; (I) mono-modal $a_{1}$; (II) mono-modal $a_{2}$; (III) bi-modal $\left(a_{1}, a_{2}\right)$; (U) unlinked towers; continuous line: stable; dashed line: unstable.
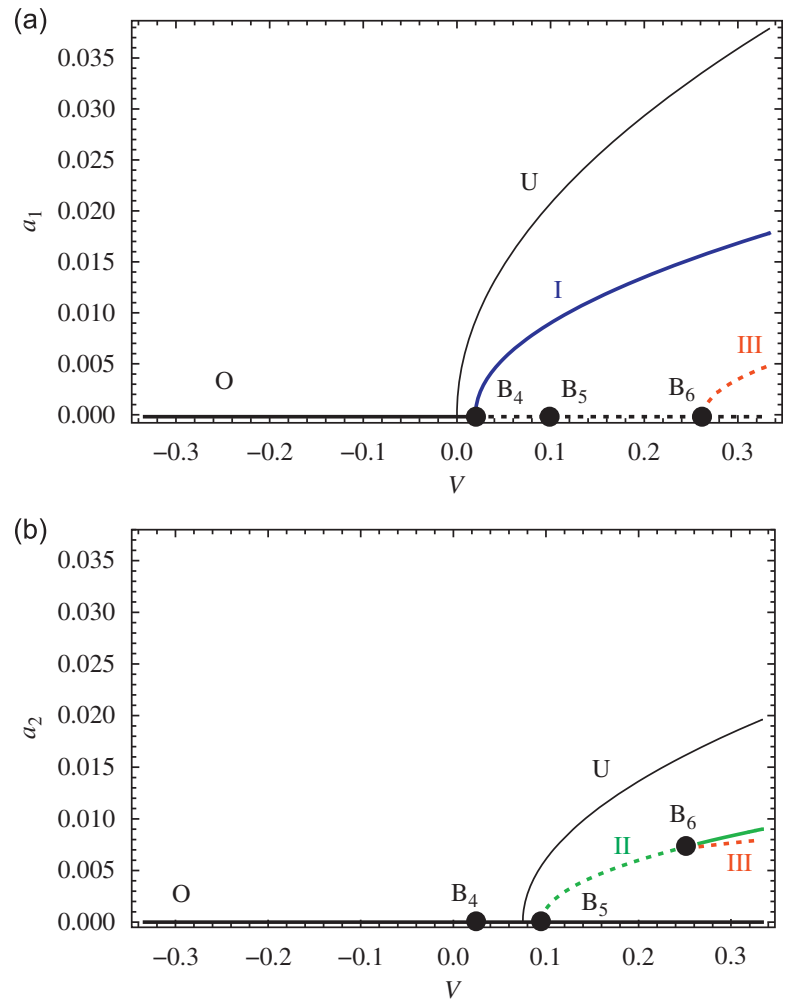

Fig. 8. Limit-cycle amplitudes $a_{1}$ (a) and $a_{2}$ (b) vs. $V$ for non-turbulent wind, very hard viscous device and $\zeta \quad-0.0003$ (path 4 in Fig. $3 \mathrm{~b}$ ). (O) Trivial; (I) mono-modal $a_{1}$; (II) mono-modal $a_{2}$; (III) bi-modal $\left(a_{1}, a_{2}\right)$; (U) unlinked towers; continuous line: stable; dashed line: unstable. 

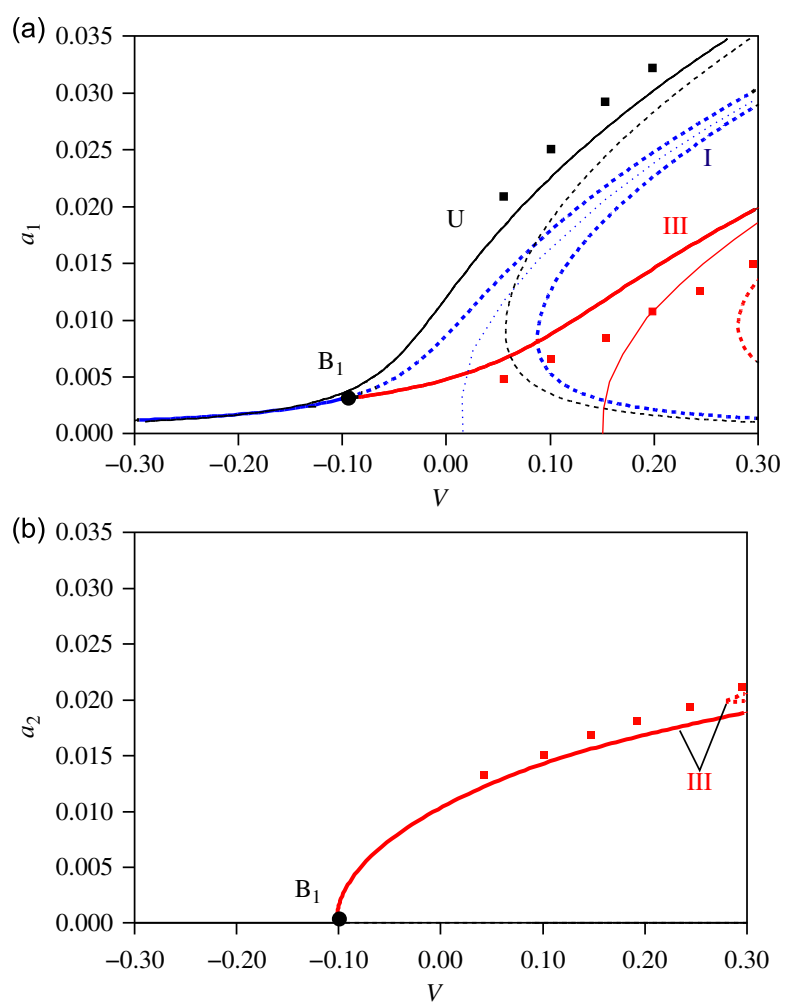

Fig. 9. Limit-cycle amplitudes $a_{1}$ (a) and $a_{2}$ (b) vs. $V$ for turbulent wind $\left(u_{1} \quad 0.033, u_{2} \quad 0\right)$, hard viscous device and $\zeta \quad 0.0005, \sigma \quad 0$. (I) Mono-modal $a_{1}$; (III) bi-modal $\left(a_{1}, a_{2}\right)$; (U) unlinked towers; continuous line: stable; dashed line: unstable, boxes: numerical integrations of the original equations (10); thin colored line: non-turbulent wind. (For interpretation of the references to color in this figure legend, the reader is referred to the web version of this article.)

value of $\zeta=0.0005$. The limit cycle amplitudes $a_{k}$ are plotted vs. the main parameter $V$ or the detuning $\sigma$. These planar bifurcation diagrams should be considered as sections of a $3 \mathrm{D}$ plot in the $\left(a_{k}, V, \sigma\right)$ space. When they are compared with Fig. 4, the effect of the turbulence is highlighted. With regard the unlinked case, in which the turbulence does not affect the right tower, the relevant $a_{2}$ response, identical to that of Figs. 4 to 8, will be omitted. All the analyses are carried out in the case of a hard viscous device.

Turbulence leading to external excitation is first considered, by taking $u_{1}=0.033, u_{2}=0$.

When the turbulence is perfectly tuned $(\sigma=0)$, the response appears as in Fig. 9. With respect to Fig. 4, the trivial solution $\mathrm{O}$ does not exist anymore, and mono modal (I) and bi modal (III) branches change as in the presence of a slight imperfection in a pitchfork bifurcation. The (perfect) solutions, relevant to non turbulent wind, are superimposed in the figures (thin lines), to highlight that they behave like backbones for the imperfect solutions. In particular, each perturbed backbone splits into two separate solutions, one on the left, the other on the right. The bifurcation point $\mathrm{B}_{1}$ indicates the origin of stable bi modal branches III, where branch I becomes unstable. It is worth noticing how the mono modal branch II does not exist anymore, but it is actually substituted by the bi modal III. Comparison with the unlinked solution, also reported in the figure, leads to the conclusion that, except for oscillations of small amplitude (on the left of the point $B_{1}$ ), the beneficial effect of the viscous device is preserved, since the amplitudes $a_{2}$ of branch III are practically overlapping branch II of Fig. 4. Again the reliability of the results obtained through the method of multiple scales is verified superimposing, in Fig. 9, also the amplitudes read on the results of direct numerical integrations on the original equations (10) (filled boxes), showing good agreement. The numerical time laws of $x(t)$ and $y(t)$ for $V=0.3$, corresponding to the stable bi modal solution III, are shown in Fig. 10, compared with the response of the unlinked left tower.

When the steady wind velocity is fixed $(V=0.3)$ and the detuning $\sigma$ is varied, the limit cycle amplitudes are shown in Fig. 11. From Figs. 9 and 11 it appears that, when a 3 D bifurcation diagram $\left(a_{k}, V, \sigma\right)$ is considered, the upper parts of branches I and III form a tube around the relevant backbone curves, as already noticed in [18]. For higher and lower values of $\sigma$, the tubes are surrounded by (not shown) amplitude periodic solutions (representing quasi periodic oscillations of the towers).

\subsection{Turbulent wind as parametric excitation}

When the turbulence acts as a parametric excitation $\left(u_{1}=0, u_{2}=0.033\right)$, the relevant bifurcation diagram appears as Fig. 12. The main effect of parametric excitation consists in splitting and shifting the non turbulent curves on the left and right sides (like a modification of stiffness in a pitchfork). Differently from the former case, the trivial solution $\mathrm{O}$ is not 

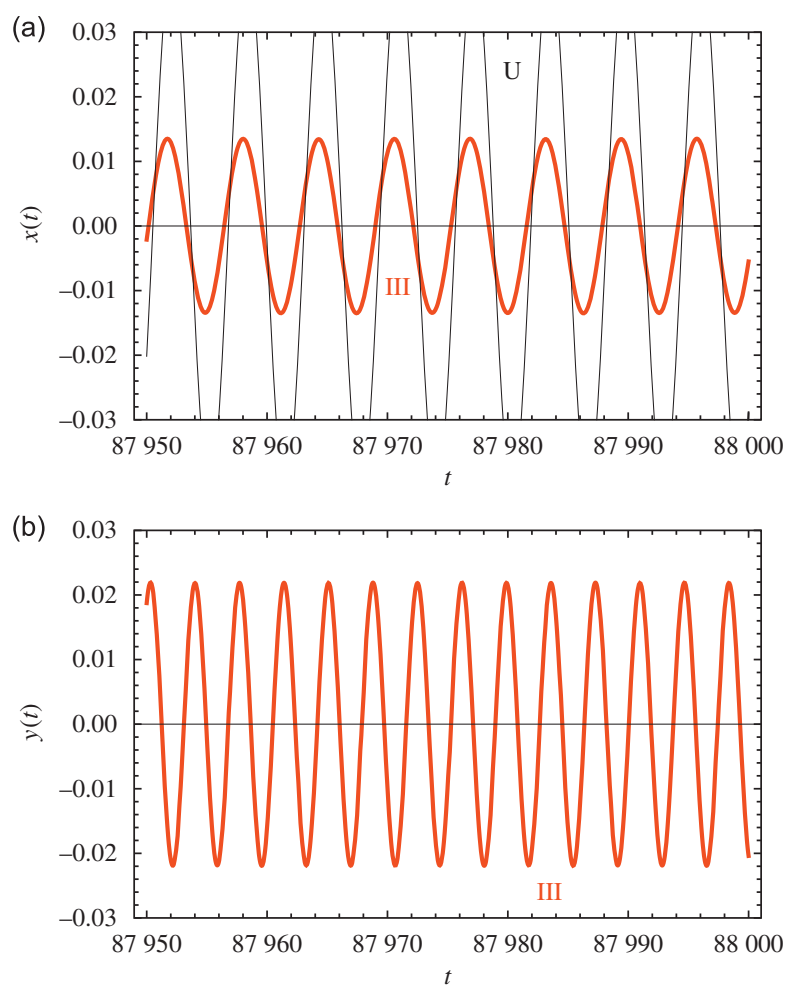

Fig. 10. Numerical integrations of Eq. (10): $x(t)(\mathrm{a})$ and $y(t)(\mathrm{b})$ for turbulent wind $\left(\begin{array}{llll}u_{1} & 0.033, u_{2} & 0\end{array}\right)$, hard viscous device and $V \quad 0.3, \zeta \quad 0.0005$. (III) Bi-modal; (U) unlinked towers.
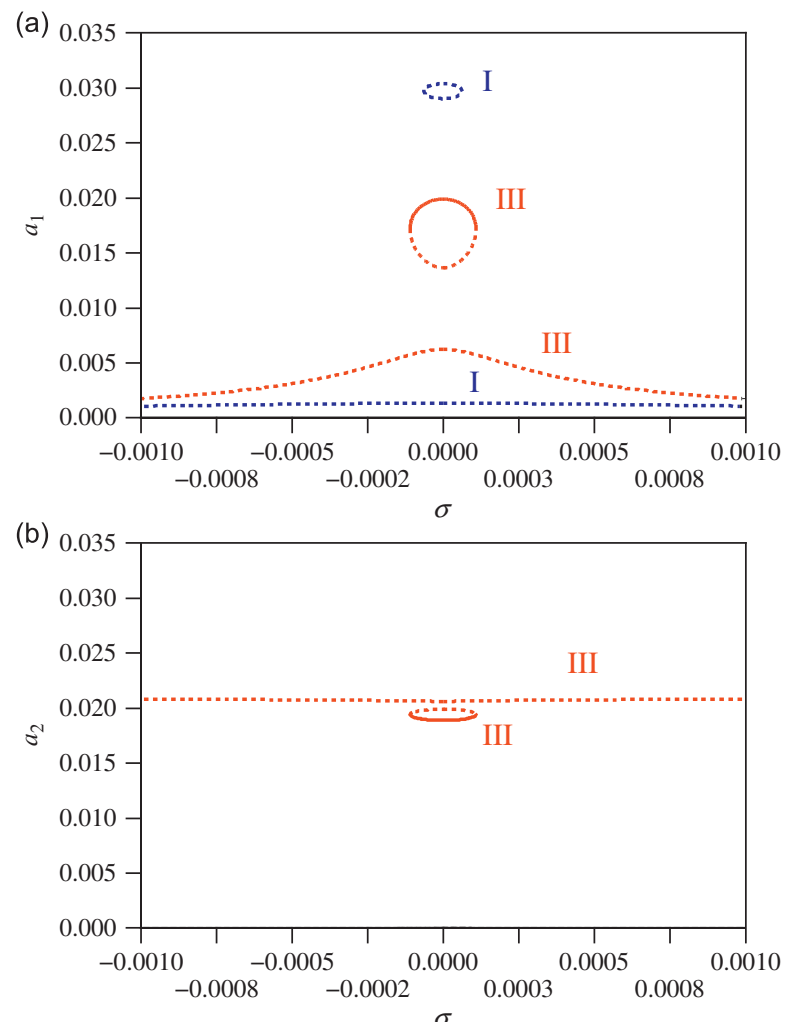

Fig. 11. Limit-cycle amplitudes $a_{1}$ (a) and $a_{2}$ (b) vs. $\sigma$ for turbulent wind $\left(u_{1}\right.$ $a_{1}$; (III) bi-modal $\left(a_{1}, a_{2}\right)$; continuous line: stable; dashed line: unstable. 

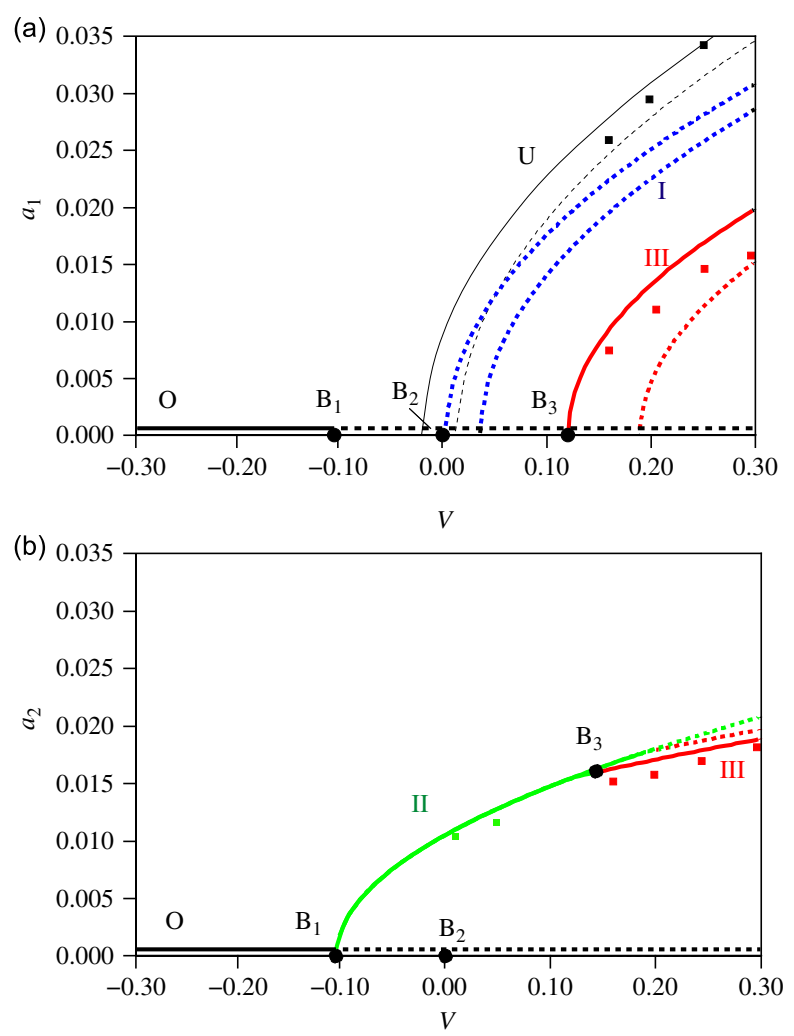

Fig. 12. Limit-cycle amplitudes $a_{1}(\mathrm{a})$ and $a_{2}$ (b) vs. $V$ for turbulent wind ( $u_{1} \quad 0, u_{2} \quad 0.033$ ), hard viscous device and $\zeta \quad 0.0005, \sigma \quad 0$. (0) Trivial; (I) mono-modal $a_{1}$; (II) mono-modal $a_{2}$; (III) bi-modal $\left(a_{1}, a_{2}\right)$; (U) unlinked towers; continuous line: stable; dashed line: unstable, boxes: numerical integrations of the original equations (10).

destroyed by the turbulence. The left branch III, which emanates from the bifurcation point $B_{3}$, is stable. Nevertheless, the actual value of $V$ at which galloping occurs is not modified by the turbulence (at $\mathrm{B}_{1}$ ), because the branch II, which is also stable, is unaffected by the parametric excitation. The beneficial effect of the viscous device is still preserved, since the amplitudes $a_{2}$ of branch II are practically the same of those of Fig. 4. Results from numerical integrations of Eq. (10) are superimposed in Fig. 12, exhibiting good agreement, while numerical time laws are shown in Fig. 13.

The limit cycles amplitude solutions are then shown vs. $\sigma$ in Fig. 14 when $V=0.3$. The steady state motions I and III again form tubes around the perfect solution, and they are surrounded by (not shown) quasi periodic motions.

\subsection{Turbulent wind as simultaneous external and parametric excitations}

When external and parametric excitations act simultaneously (see Fig. 15, relevant to $u_{1}=u_{2}=0.033$ ), a new mono modal branch appears, for high values of $V$, compared with the purely external excitation case. Actually, it is a loop of branch I, as it is highlighted by the section of the $3 \mathrm{D}$ plot taken for fixed $V=0.3$ (see Fig. 17). This behavior also occurs for the unlinked tower and, in general, for 1 d.o.f systems [18,22]. In the present problem, however, a second loop also appears in the bi modal branch III, which did not appear in Fig. 15 because it is not intersected by the plane $\sigma=0$. Outside the tube, quasi periodic motions (not shown) are found. As in the previous case, solution III substitutes solution II, which disappears. Numerical results from direct integrations of Eq. (10) are also shown in Fig. 15, while in Fig. 16 the time laws of $x(t)$ and $y(t)$ are reported. The viscous device again mitigates the oscillations of the unlinked tower.

\section{Conclusions}

In this paper, a 2 d.o.f. nonlinear dynamic system, representative of two planar towers, linked by a nonlinear viscous device, hit by turbulent wind flow acting orthogonally, has been studied. The wind causes simultaneous self , external and parametric excitations, the first due to the steady part of the aerodynamic force, the last two due to the turbulent part of the wind. The Multiple Scale Method has been used to obtain Amplitude Modulation Equations, under (a) 1:1 resonance condition between the fundamental component of the turbulent wind and one of the two towers, and (b) in no internal resonance conditions.

The dynamic behavior of the system, consequent to different kinds of excitations, analyzed separately, has been investigated in the space of the bifurcation parameters. Limit cycles involving just one (mono modal) or two (bi modal) 

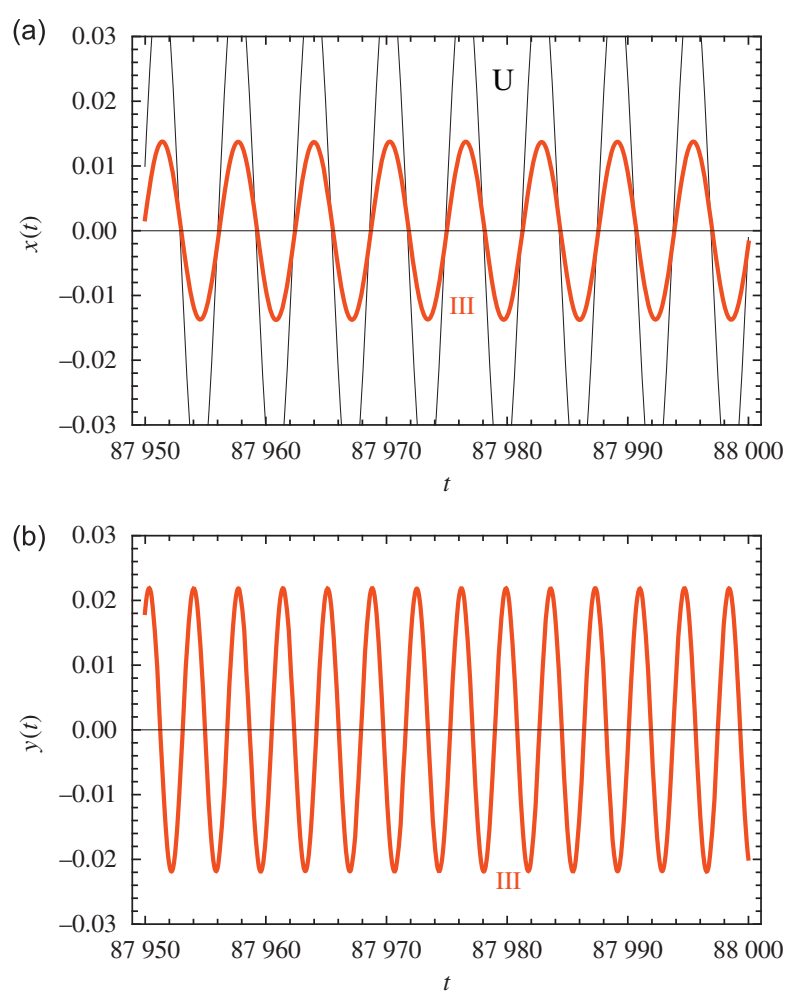

Fig. 13. Numerical integrations of Eq. (10): $x(t)$ (a) and $y(t)$ (b) for turbulent wind $\left(\begin{array}{llll}u_{1} & 0, u_{2} & 0.033\end{array}\right)$, hard viscous device and $V \quad 0.3$, $\zeta \quad 0.0005$. (III) Bi-modal; (U) unlinked towers.
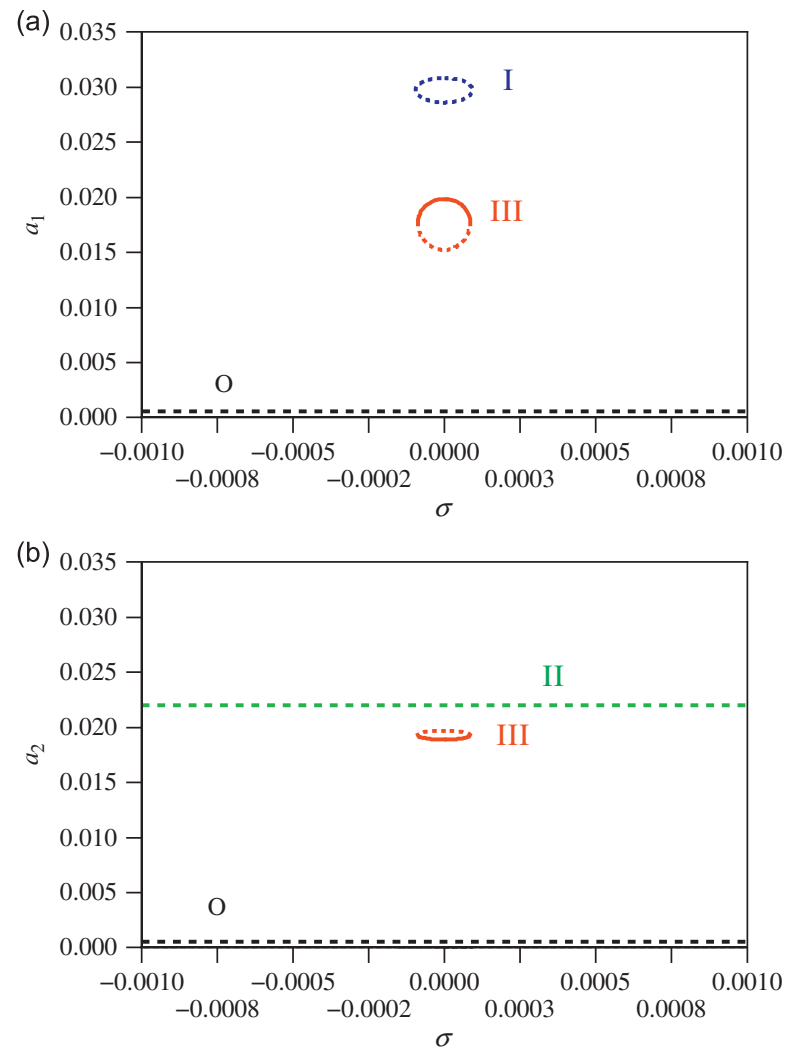

Fig. 14. Limit-cycle amplitudes $a_{1}$ (a) and $a_{2}$ (b) vs. $\sigma$ for turbulent wind $\left(u_{1} \quad 0, u_{2} \quad 0.033\right.$ ), hard viscous device and $\zeta \quad 0.0005, V \quad 0.3$. (0) Trivial; (I) mono-modal $a_{1}$; (II) mono-modal $a_{2}$; (III) bi-modal $\left(a_{1}, a_{2}\right)$; continuous line: stable; dashed line: unstable. 
(a)
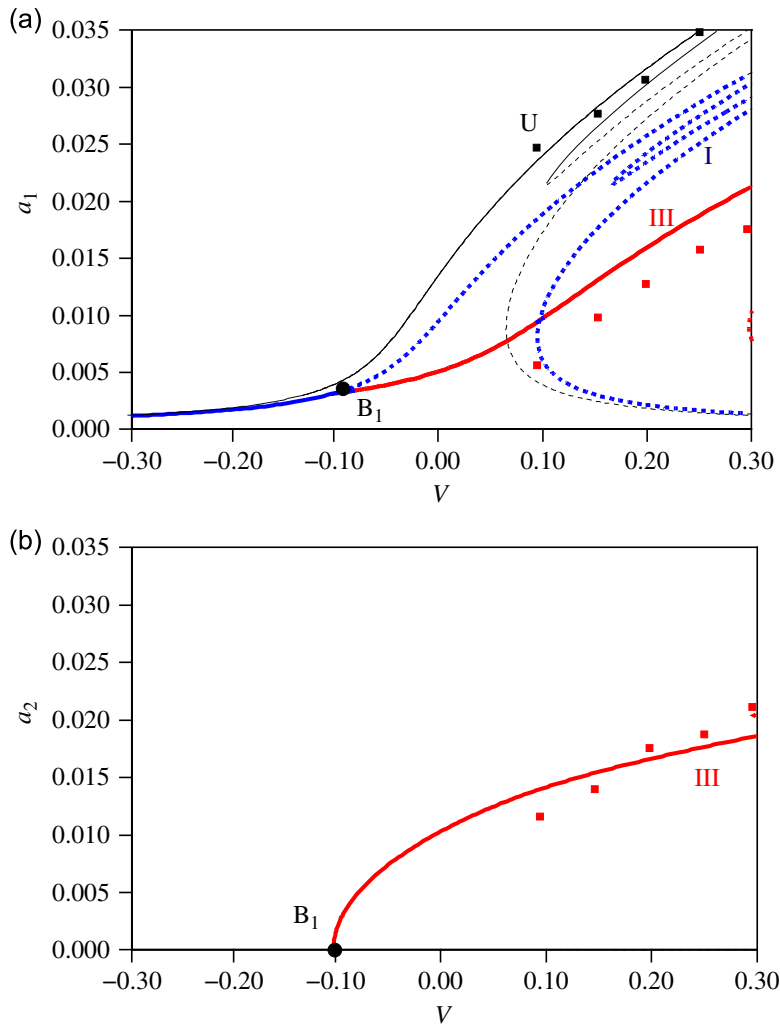

Fig. 15. Limit-cycle amplitudes $a_{1}$ (a) and $a_{2}$ (b) vs. $V$ for turbulent wind ( $u_{1} \quad u_{2} \quad 0.033$ ), hard viscous device and $\zeta \quad 0.0005, \sigma \quad 0$. (I) Mono-modal $a_{1}$; (III) bi-modal $\left(a_{1}, a_{2}\right)$; (U) unlinked towers; continuous line: stable; dashed line: unstable, boxes: numerical integrations of the original equations (10).
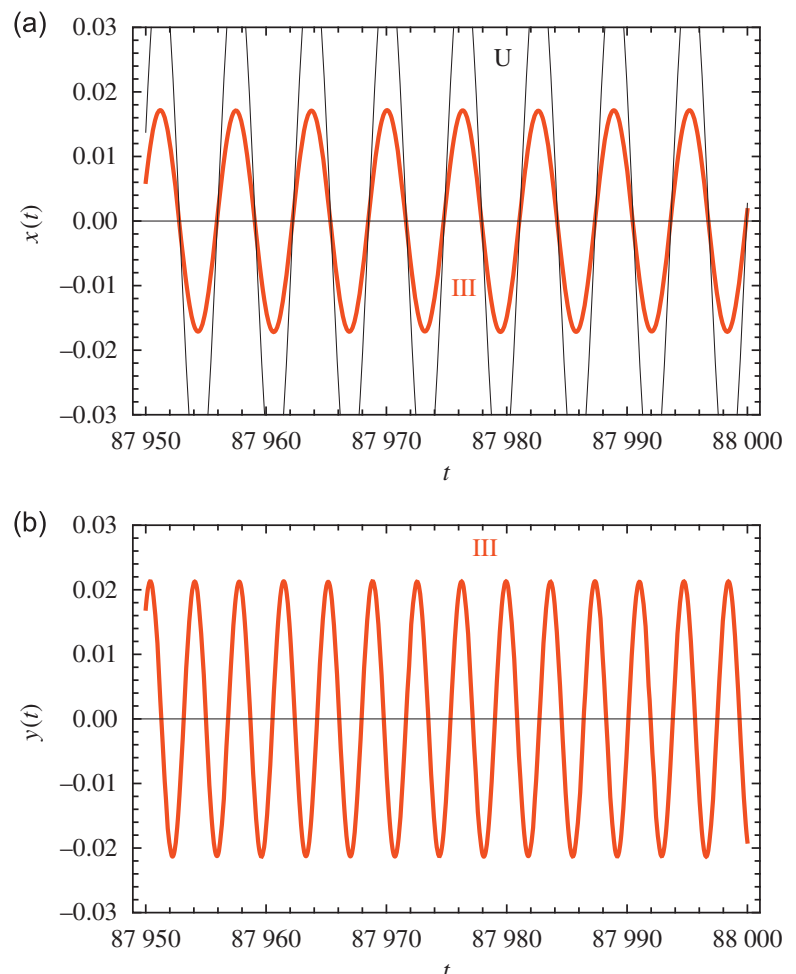

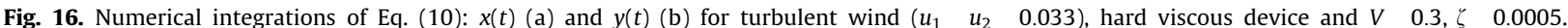
(III) Bi-modal; (U) unlinked towers. 

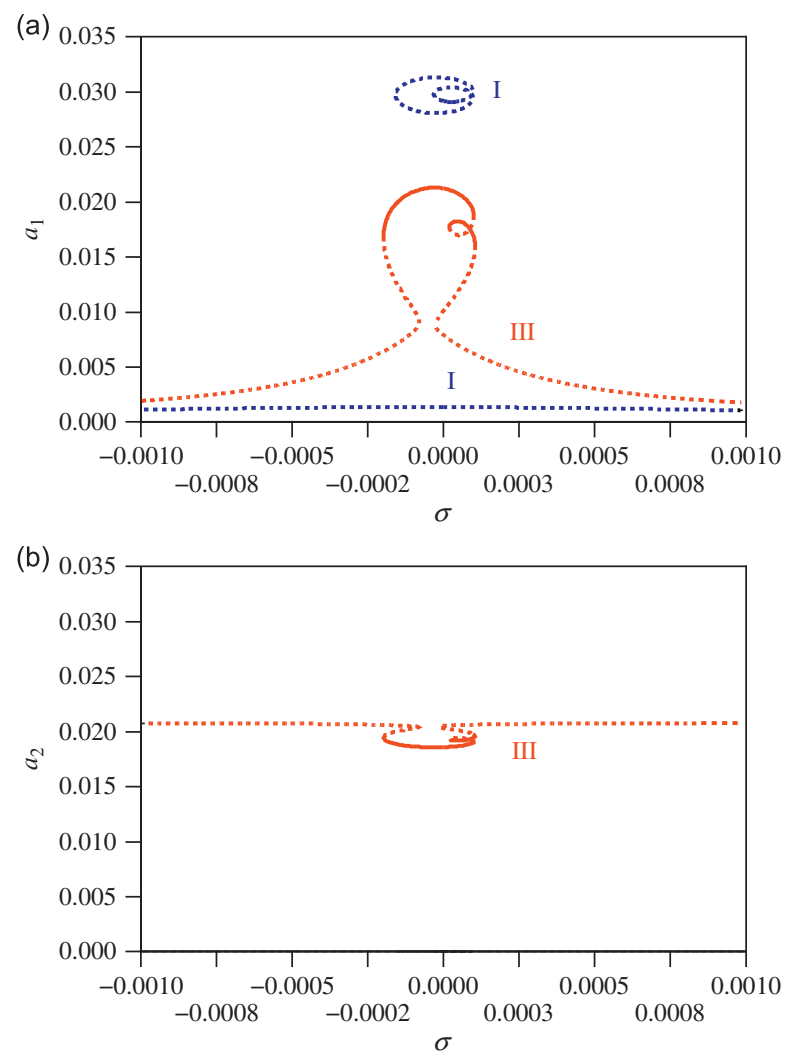

Fig. 17. Limit-cycle amplitudes $a_{1}$ (a) and $a_{2}$ (b) vs. $\sigma$ for turbulent wind ( $u_{1} \quad u_{2} \quad$ 0.033), hard viscous device and $\zeta \quad 0.0005, V \quad 0.3$. (I) Mono-modal $a_{1}$; (III) bi-modal $\left(a_{1}, a_{2}\right)$; continuous line: stable; dashed line: unstable.

components have been detected, and their amplitude and stability evaluated. In particular, the non resonant double Hopf bifurcation scenario, known in the literature, has been recovered for non turbulent wind. The following conclusions have been obtained.

1. The viscous device increases the galloping wind velocity; moreover, hard nonlinear viscosity mitigates the amplitude of the limit cycle, with beneficial effects which increase with the magnitude of nonlinearity.

2. When the turbulent wind produces external excitation only, it works as an imperfection for the self excitation case, leading to limit cycle amplitude close to the previous ones. When turbulence causes parametric excitation only, it splits and moves apart the self excitation branches.

3. When external and parametric excitations coexist, some loops in branches already observed in the literature for 1 d.o.f. systems, are found to occur also in bi modal branches.

4. In all the studied cases of turbulent wind, when the two tower system is compared with the single tower system, the viscous device is found to reduce the critical wind velocity at which the trivial solution loses stability and to reduce the amplitude of the stable limit cycles. Therefore it represents an efficient passive controller against wind induced instability phenomena.

Appendix A. Notation The following list contains the symbols used in this paper, with corresponding meaning and unit: 
total height of the towers $(\mathrm{m})$

mass per unit length of the $i$-th tower $(\mathrm{kg} / \mathrm{m})$

$n_{i}$ aerodynamic load linear density of the $i$-th tower $(\mathrm{N} / \mathrm{m})$ load linear density of the $i$-th tower $(\mathrm{N} / \mathrm{m})$

curvilinear abscissa of the rods (m)

non-dimensional curvilinear abscissa of the rods

shear force of the $i$-th tower $(\mathrm{N})$

time $(s)$

non-dimensional time

time scales (nondim.)

wind velocity $(\mathrm{m} / \mathrm{s})$

average wind velocity $(\mathrm{m} / \mathrm{s})$

non-dimensional average wind velocity

critical average wind velocity $(\mathrm{m} / \mathrm{s})$

non-dimensional critical average wind velocity of the left rod

non-dimensional critical average wind velocity of the right rod

turbulent wind velocity $(\mathrm{m} / \mathrm{s})$

amplitudes of the turbulent wind velocity components (nondim.)

non-dimensional turbulent wind velocity

mean wind velocity increment (nondim.)

critical increments of the wind velocity of the $i$-th rod (nondim.)

displacement of the $j$-th floor of the left tower ( $\mathrm{m}$ )

displacement of the $k$-th floor of the right tower $(\mathrm{m})$

modal displacement of the left rod (nondim.)

rescaled variable $x$ (nondim.)

series expansion terms for $\hat{x}$ (nondim.)

modal displacement of the right rod (nondim.)

rescaled variable $y$ (nondim.)

series expansion terms for $\hat{y}$ (nondim.)

mass ratio (nondim.)

cross-section side ratio (nondim.)

relative velocity of the tips of the two rods $(\mathrm{m} / \mathrm{s})$

perturbation parameter (nondim.)

damping splitting parameter (nondim.)

internal damping coefficient of the $i$-th tower ( $\mathrm{N} \mathrm{s}$ )

phase of the first-order solution of the $i$-th rod (nondim.)

linear and cubic coefficients of the viscous device $\left(\mathrm{kg} / \mathrm{s}, \mathrm{kg} \mathrm{s} / \mathrm{m}^{2}\right)$

$j$-th eigenvalue of the Jacobian matrix (nondim.)

characteristic parameter of the viscous device (nondim.)

critical modal damping ratio of the right rod (nondim.)

modal damping ratio of the $i$-th rod (nondim.)

air mass density $\left(\mathrm{kg} / \mathrm{m}^{3}\right)$

frequency detuning parameter (nondim.)

first modal shape of the $i$-th rod (nondim.)

redefinition of the phase of the first order solution of the $i$-th rod (nondim.)

fundamental forcing frequency of the turbulent component (nondim.)

critical forcing frequency (nondim.)

natural circular frequency of the $i$-th $\operatorname{rod}(\mathrm{rad} / \mathrm{s})$

natural circular frequency ratio (nondim.)

\section{References}

[1] J. Guckenheimer, P. Holmes, Nonlinear Oscillations, Dynamical Systems, and Bifurcation of Vector Fields, Springer-Verlag, New York, 1983.

[2] G.R. Itovich, J.L. Moiola, Non-resonant double Hopf bifurcations: the complex case, Journal of Sound and Vibration 322 (2009) 358-380.

[3] P. Yu, Analysis on double Hopf bifurcation using computer algebra with the aid of multiple scales, Nonlinear Dynamics 27 (2002) 19-53.

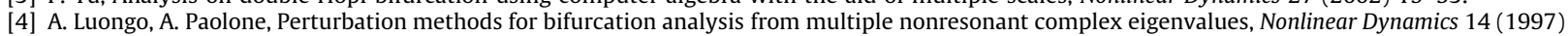
$193-210$

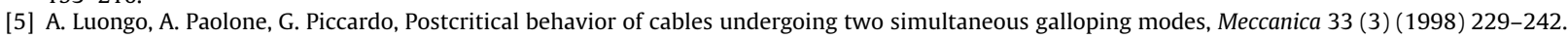

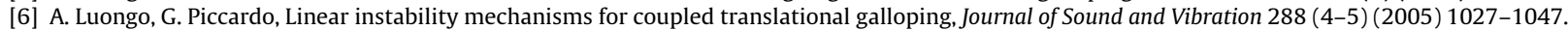

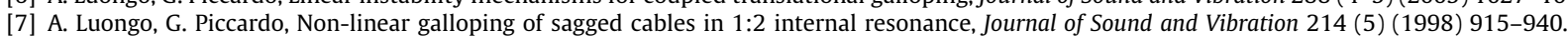

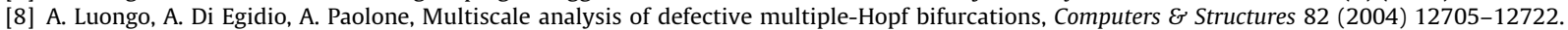

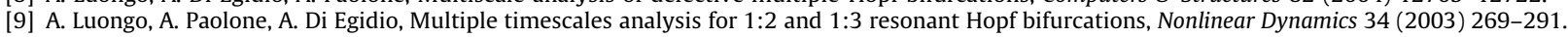

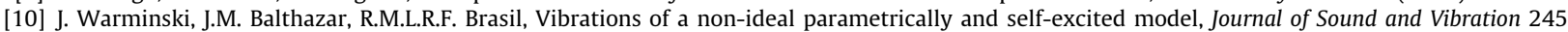
(2001) 363-374. 
[11] A. Tondl, H. Ecker, On the problem of self-excited vibration quenching by means of parametric excitation, Archive of Applied Mechanics 72 (2003) 923-932.

[12] A. Tondl, R. Nabergoj, The effect of parametric excitation on a self-excited three-mass system, International Journal of Non-Linear Mechanics 39 (2004) $821-832$.

[13] K.R. Asfar, A.H. Nayfeh, D.T. Mook, Response of self-excited two-degree-of-freedom systems to multifrequency excitations, Journal of Sound and Vibration 84 (1982) 199-221.

[14] K. Szabelski, J. Warminski, Vibration of a non-linear self-excited system with two degrees of freedom under external and parametric excitation, Nonlinear Dynamics 14 (1997) 23-36.

[15] J. Warminski, Nonlinear normal modes of a self-excited system driven by parametric and external excitations, Nonlinear Dynamics 61 (4) (2010) 677-689.

[16] A. Luongo, D. Zulli, Dynamic instability of inclined cables under combined wind flow and support motion, Nonlinear Dynamics doi:10.1007/ s11071-011-9958-9.

[17] M. Abdel-Rohman, Effect of unsteady wind flow on galloping of tall prismatic structures, Nonlinear Dynamics 26 (2001) $231-252$.

[18] A. Luongo, D. Zulli, Parametric, external and self-excitation of a tower under turbulent wind flow, Journal of Sound and Vibration 330 (13) 3057-3069.

[19] A.H. Nayfeh, D.T. Mook, Nonlinear Oscillations, John Wiley, New York, 1979.

[20] A. Luongo, G. Rega, F. Vestroni, On nonlinear dynamics of planar shear indeformable beams, Journal of Applied Mechanics 53 (1986) 619-624.

[21] M. Novak, Aeroelastic galloping of prismatic bodies, Journal of Engineering Mechanics 95 (EM1) (1969) 115-141.

[22] K. Szabelski, J. Warminski, Parametric self-excited non-linear system vibrations analysis with inertial excitation, International Journal of Non-Linear Mechanics 30 (1995) 179-189. 